\title{
Possible configurations of the magnetic field in the outer magnetosphere during geomagnetic polarity reversals
}

\author{
D. M. Willis ${ }^{1, *}$, A. C. Holder ${ }^{2,3}$, C. J. Davis ${ }^{2}$ \\ ${ }^{1}$ Space and Astrophysics Group, Department of Physics, University of Warwick, Coventry CV4 7AL, UK \\ E-mail: dave@mail.eiscat.rl.ac.uk \\ ${ }^{2}$ Rutherford Appleton Laboratory, Chilton, Didcot, Oxon OX11 0QX, UK \\ ${ }^{3}$ School of Mathematical and Information Sciences, Coventry University, Coventry CV1 5FB, UK
}

Received: 8 February 1999 / Revised: 9 July 1999 / Accepted: 20 July 1999

\begin{abstract}
Possible configurations of the magnetic field in the outer magnetosphere during geomagnetic polarity reversals are investigated by considering the idealized problem of a magnetic multipole of order $m$ and degree $n$ located at the centre of a spherical cavity surrounded by a boundless perfect diamagnetic medium. In this illustrative idealization, the fixed spherical (magnetopause) boundary layer behaves as a perfectly conducting surface that shields the external diamagnetic medium from the compressed multipole magnetic field, which is therefore confined within the spherical cavity. For a general magnetic multipole of degree $n$, the non-radial components of magnetic induction just inside the magnetopause are increased by the factor $\{1+[(n+1) / n]\}$ relative to their corresponding values in the absence of the perfectly conducting spherical magnetopause. An exact equation is derived for the magnetic field lines of an individual zonal $(m=0)$, or axisymmetric, magnetic multipole of arbitrary degree $n$ located at the centre of the magnetospheric cavity. For such a zonal magnetic multipole, there are always two neutral points and $n-1$ neutral rings on the spherical magnetopause surface. The two neutral points are located at the poles of the spherical magnetopause. If $n$ is even, one of the neutral rings is coincident with the equator; otherwise, the neutral rings are located symmetrically with respect to the equator. The actual existence of idealized higher-degree $(n>1)$ axisymmetric magnetospheres would necessarily imply multiple $(n+1)$ magnetospheric cusps and multiple $(n)$ ring currents. Exact equations are also derived for the magnetic field lines of an individual non-axisymmetric magnetic multipole, confined by a perfectly conducting spherical magnetopause, in two special cases; namely, a symmetric sectorial multipole $(m=n)$ and an antisymmetric sectorial multipole $(m=n-1)$. For both these
\end{abstract}

Correspondence to: D.M. Willis

* Also Honorary Research Associate, Rutherford Appleton Laboratory, Chilton, Didcot, Oxon OX11 0QX, UK and Visiting Reader in Physics, University of Sussex, Falmer, Brighton BN1 9QH, UK non-axisymmetric magnetic multipoles, there exists on the spherical magnetopause surface a set of neutral points linked by a network of magnetic field lines. Novel magnetospheric processes are likely to arise from the existence of magnetic neutral lines that extend from the magnetopause to the surface of the Earth. Finally, magnetic field lines that are confined to, or perpendicular to, either special meridional planes or the equatorial plane, when the multipole is in free space, continue to be confined to, or perpendicular to, these same planes when the perfectly conducting magnetopause is present.

Key words. Geomagnetism and paleomagnetism (reversals-process, time scale, magnetostratigraphy) Magnetospheric physics (magnetopause, cusp, and boundary layers; magnetospheric configuration and dynamics)

\section{Introduction}

An idealized model is developed to elucidate the possible configurations of the magnetic field in the outer magnetosphere during geomagnetic polarity reversals. Possible magnetic-field configurations in the inner magnetosphere have been considered in previous papers (Willis and Young, 1987; Willis and Gardiner, 1988). These papers have been based on the assumption that, during a geomagnetic polarity reversal, the transitional magnetic field can sometimes be represented approximately by a single, non-dipolar, magnetic multipole of order $m$ and degree $n$. In particular, Willis and Young (1987) derived an exact equation for the magnetic field lines of an individual axisymmetric (or zonal) magnetic multipole of arbitrary degree $(n)$. Subsequently, Jeffreys (1988) presented an alternative and somewhat simpler mathematical derivation of the equation for the field lines of a single axisymmetric magnetic multipole. This result was then generalized to the case of an arbitrary 
linear combination of axisymmetric magnetic multipoles by Backus (1988), who showed that an exact equation for the magnetic field lines can be obtained elegantly by analogy with the solution of an equivalent problem in hydrodynamics (Lamb, 1945). In a special extension to non-axisymmetric magnetic fields, Willis and Gardiner (1988) derived exact equations for the magnetic field lines of both symmetric sectorial $(m=n)$ and antisymmetric sectorial $(m=n-1)$ individual magnetic multipoles of arbitrary degree $(n)$.

The early palaeomagnetic evidence supporting the belief that the transitional magnetic field in the inner magnetosphere can sometimes be represented approximately by a single, non-dipolar, magnetic multipole has been summarized in previous papers (Willis and Young, 1987; Willis and Gardiner, 1988; Willis et al., 1997). In a recent review paper, Merrill and McFadden (1999) have concluded that existing palaeomagnetic data are inadequate to determine conclusively whether the transitional magnetic field is predominantly dipolar or non-dipolar at the Earth's surface. Nevertheless, the available evidence suggests that non-axisymmetric multipoles dominate the transitional magnetic field. Any major change in the configuration of the geomagnetic field during a polarity reversal (or even a large excursion) would inevitably lead to dramatic changes in geomagnetically trapped radiation, the geographical distribution of precipitating auroral particles and the geographical distribution of cosmic rays impinging on the Earth's upper atmosphere.

The configuration of the magnetic field in the outer magnetosphere during a geomagnetic polarity reversal (or major excursion) is simulated in the present investigation by placing a magnetic multipole (of arbitrary order $m$ and degree $n$ ) at the centre of an ideal, perfectly conducting, spherical magnetopause. Stated alternatively, a magnetic multipole is placed at the centre of a spherical cavity surrounded by a boundless perfect diamagnetic medium, as illustrated schematically in Fig. 1. This approach is similar to that adopted by $\mathrm{Wu}$ and Cole $(1984 a, b)$ in their formulation of a "new" iterative method of calculating the shape of the actual elongated magnetopause, which separates the flowing solar-wind plasma in the magnetosheath from the confined geomagnetic field in the magnetosphere. In an appendix, Wu and Cole (1984a) derived an analytic solution for the particular case of a magnetic dipole located at the centre of a spherical cavity surrounded by a boundless perfect diamagnetic medium. These authors used this analytic solution to test their "new" iterative numerical method of solving the integral equation for the magnetic field just inside a spherical magnetopause. Subsequently, Wu and Cole (1984b) successfully tested their "new" iterative numerical method of determining the shape of the magnetopause in the special situation for which the external (magnetosheath) plasma pressure is assumed to be constant and homogeneous over the entire magnetopause.

The theoretical treatment presented here provides an exact analytic solution for the magnetic field inside an idealized spherical magnetosphere, which results from

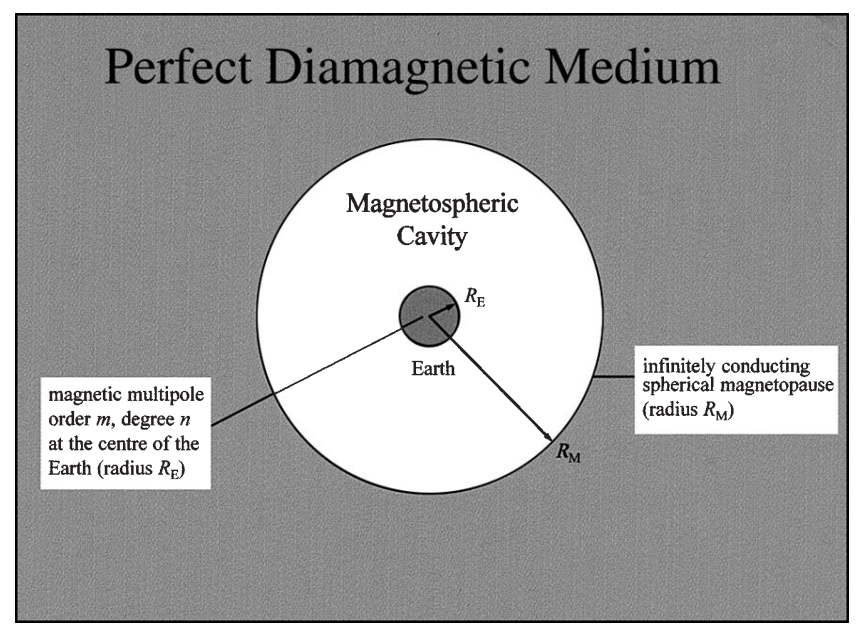

Fig. 1. Schematic illustration of a magnetic multipole (of arbitrary order $m$ and degree $n$ ) located at the centre of a spherical (magnetosphere) cavity within a boundless perfect diamagnetic medium. The fixed spherical (magnetopause) boundary layer behaves as a perfectly conducting surface that shields the external diamagnetic medium from the compressed multipole magnetic field within the spherical cavity

the confinement of a multipolar transitional magnetic field by a perfectly conducting spherical magnetopause. Although the actual magnetopause is almost certainly not spherical, the idealized problem considered here provides considerable physical insight into the various possible topologies of the magnetic-field configuration in the outer magnetosphere during geomagnetic polarity reversals. In particular, the magnetic-field configuration at the spherical magnetopause itself, including the distribution of neutral points and neutral rings, is topologically similar to that arising for the classical (Chapman-Ferraro) magnetopause boundary. This classical magnetopause boundary is based on the assumption of a unidirectional stream of cold solar-wind particles being specularly reflected at a free magnetopause surface (Beard, 1964, 1967). It should be stressed, however, that no attempt is made here to consider the merging (or "reconnection") of the interplanetary and terrestrial magnetic fields at the magnetopause.

\section{The magnetic field in an idealized spherical magnetosphere}

It is convenient to derive the magnetic field in the idealized spherical magnetosphere by considering the confinement of the general spherical harmonic expansion of the Earth's main magnetic field by a perfectly conducting spherical magnetopause. The general approach adopted in Sect. 2.1 facilitates comparisons with the results presented in previous papers (Willis and Young, 1987; Willis and Gardiner, 1988; Willis et al., 1997). As indicated in Fig. 1, the mean radius of the Earth is denoted by $R_{\mathrm{E}}$ and the radius of the concentric spherical magnetopause is denoted by $R_{\mathrm{M}}$. The main emphasis of this study is on the confinement of an individual magnetic multipole, of specified degree $n$ and 
order $m$, by a perfectly conducting spherical magnetopause. Nevertheless, a more general result is presented in Sect. 2.2 for the magnetic-field components within the magnetosphere in the case of an arbitrary linear combination of multipoles of identical degree $n$ but varying order $m$.

\subsection{Spherical harmonic analysis of the magnetospheric magnetic field}

Referred to a system of spherical polar coordinates $(r, \theta, \phi)$, the general solution of Laplace's equation $\left(\nabla^{2} V=0\right)$ for the magnetic scalar potential $(V)$ in the magnetosphere can be expressed in the form (Chapman and Bartels, 1940; Price, 1967; Vestine, 1967; Wu and Cole, 1984a):

$$
\begin{aligned}
V= & \sum_{n=1}^{\infty} \sum_{m=0}^{n}\left[\left(A_{n}^{m} r^{n}+B_{n}^{m} r^{-n-1}\right) \cos m \phi\right. \\
& \left.+\left(C_{n}^{m} r^{n}+D_{n}^{m} r^{-n-1}\right) \sin m \phi\right] P_{n}^{m}(\cos \theta),
\end{aligned}
$$

where $A_{n}^{m}, B_{n}^{m}, C_{n}^{m}$ and $D_{n}^{m}$ are arbitrary coefficients, or constants, to be determined from the boundary conditions. The pairs of coefficients $A_{n}^{m}, C_{n}^{m}$ and $B_{n}^{m}, D_{n}^{m}$ refer, respectively, to contributions that originate from magnetic sources that are external $\left(r>R_{\mathrm{E}}\right)$ and internal $\left(r<R_{\mathrm{E}}\right)$ with respect to the surface of the Earth (mean radius $R_{\mathrm{E}}$ ). The other quantities appearing in Eq. (1) are defined in the following paragraph.

At points close to the Earth and remote from the magnetopause (i.e. $R_{\mathrm{E}} \leq r \ll R_{\mathrm{M}}$ ), the magnetospheric magnetic field is dominated by the Earth's main magnetic field. The external $\left(r \geq R_{\mathrm{E}}\right)$ magnetic scalar potential $\left(V^{i}\right)$ of this geomagnetic field of internal origin is normally expressed in the form (Chapman and Bartels, 1940; Roederer, 1972; Willis and Young, 1987):

$$
\begin{aligned}
V^{i}= & \sum_{n=1}^{\infty} \sum_{m=0}^{n} R_{\mathrm{E}}\left(R_{\mathrm{E}} / r\right)^{n+1} \\
& \times\left(g_{n}^{m} \cos m \phi+h_{n}^{m} \sin m \phi\right) P_{n}^{m}(\cos \theta),
\end{aligned}
$$

where the superscript $i$ is used to signify that this contribution is of internal origin. In this representation of the main geomagnetic field, positions on the surface of the Earth, and in the magnetosphere, are specified in terms of (geographic) spherical polar coordinates $(r, \theta, \phi)$ with origin $\mathrm{O}$ at the centre of the Earth. These coordinates are defined as follows: $r$ is the radial distance $\left(r \geq R_{\mathrm{E}}\right) ; \theta$ is the geographic co-latitude with the north geographic pole at $\theta=0$; and $\phi$ is the geographic longitude measured east of Greenwich. The radius of the reference sphere, $r=R_{\mathrm{E}}$, is taken to be the mean radius of the Earth $(6371.2 \mathrm{~km}) ; P_{n}^{m}(\cos \theta)$ is Schmidt's partially (or quasi-) normalized associated Legendre function of order $m$ and degree $n$ (where $m$ and $n$ are integers); $g_{n}^{m}$ and $h_{n}^{m}$ are the experimentally determined spherical harmonic (or Gauss, or Schmidt) coefficients for the particular epoch considered; and all physical quantities are measured in SI units. Our definition of scalar magnetic potential $(V)$ is such that the spherical harmonic coefficients $g_{n}^{m}$ and $h_{n}^{m}$ have the dimensions of magnetic induction (i.e. $\mathbf{B}=-\operatorname{grad} V$ ).

The spherical harmonic expansion defined by Eq. (2) is valid only outside the region of origin of the Earth's main magnetic field, which comprises the solid inner core and the liquid outer core, in an ideal external region containing no sources of magnetic field (i.e. curl $\mathbf{B}=0$ ). In the present context, Eq. (2) represents the scalar potential of the Earth's main magnetic field in the ideal external region $\left(R_{\mathrm{E}} \leq r \leq R_{\mathrm{M}}\right)$. At the spherical boundary, $r=R_{\mathrm{M}}$, the radial component of the total magnetic field $\left(B_{r}\right)$ must vanish because the magnetopause is assumed to be perfectly conducting or, stated alternatively, because the boundless surrounding medium $\left(R_{\mathrm{M}}<r<\infty\right)$ is assumed to be perfectly diamagnetic. The mathematical boundary conditions then become $V \rightarrow V^{i}$ as $r \rightarrow 0$ and $B_{r}=-\partial V / \partial r=0$ at $r=R_{\mathrm{M}}$. Applying these two boundary conditions to Eqs. (1) and (2) yields the following relations for the coefficients $A_{n}^{m}, B_{n}^{m}, C_{n}^{m}$ and $D_{n}^{m}$ :

$A_{n}^{m}=\frac{(n+1)}{n} \frac{R_{\mathrm{E}}^{n+2}}{R_{\mathrm{M}}^{2 n+1}} g_{n}^{m}, \quad C_{n}^{m}=\frac{(n+1)}{n} \frac{R_{\mathrm{E}}^{n+2}}{R_{\mathrm{M}}^{2 n+1}} h_{n}^{m}$,

$B_{n}^{m}=R_{\mathrm{E}}^{n+2} g_{n}^{m}, \quad D_{n}^{m}=R_{\mathrm{E}}^{n+2} h_{n}^{m}$.

If these values of the coefficients are substituted back into Eq. (1), the expression for the total magnetic scalar potential in the magnetosphere $\left(R_{\mathrm{E}} \leq r \leq R_{\mathrm{M}}\right)$ becomes

$$
\begin{aligned}
V= & \sum_{n=1}^{\infty} \sum_{m=0}^{n} R_{\mathrm{E}}\left(R_{\mathrm{E}} / r\right)^{n+1}\left\{1+[(n+1) / n]\left(r / R_{\mathrm{M}}\right)^{2 n+1}\right\} \\
& \times\left(g_{n}^{m} \cos m \phi+h_{n}^{m} \sin m \phi\right) P_{n}^{m}(\cos \theta) .
\end{aligned}
$$

As the radius of the spherical magnetopause becomes infinitely large (i.e. $R_{\mathrm{M}} \rightarrow \infty$ or, equally, $R_{\mathrm{M}} / R_{\mathrm{E}} \rightarrow \infty$ ), Eq. (5) reduces to Eq. (2), which correctly confirms that the magnetic field in an unbounded magnetosphere results solely from the Earth's main magnetic field of internal origin. Therefore, every magnetic-field component (and hence every field-line equation) presented here reduces to the correct limiting form as $R_{\mathrm{M}} \rightarrow \infty$, as can be verified by comparing results presented here with the corresponding results presented in the papers by Willis and Young (1987) and Willis and Gardiner (1988). No allowance is made in this initial investigation, however, for magnetic fields that might result from possible distributions of charged particles "trapped" in such an idealized spherical magnetosphere.

\subsection{The magnetic-field components in the magnetosphere}

It is shown succinctly in Appendix A that for a general magnetic multipole of degree $n$ (and not just an individual zonal, symmetric sectorial or antisymmetric sectorial multipole) the magnetic-field components $\left(B_{r, n}, B_{\theta, n}, B_{\phi, n}\right)$ in the magnetosphere can be expressed in the following form:

$\tilde{B}_{r, n}=\left[1-\left(r / R_{\mathrm{M}}\right)^{2 n+1}\right] B_{r, n}$, 
$\tilde{B}_{\theta, n}=\left\{1+[(n+1) / n]\left(r / R_{\mathrm{M}}\right)^{2 n+1}\right\} B_{\theta, n}$,

$\tilde{B}_{\phi, n}=\left\{1+[(n+1) / n]\left(r / R_{\mathrm{M}}\right)^{2 n+1}\right\} B_{\phi, n}$,

where the presence (absence) of the tilde signifies the presence (absence) of the perfectly conducting spherical magnetopause. Equations (6), (7) and (8) are valid for a magnetic multipole having a magnetic scalar potential $\left(\tilde{V}_{n}\right)$ identical to that obtained by omitting the summation over $n$ in Eq. (5) but retaining the summation over $m$.

Therefore, Eqs. (6), (7) and (8) are valid for a general magnetic multipole of degree $n$, defined by an arbitrary linear combination of individual multipoles having the same degree $n$ but different orders $m(0 \leq m \leq n)$. Equation (6) confirms that the radial component of magnetic induction just inside the spherical magnetopause $\left(r=R_{\mathrm{M}}\right)$ is reduced to zero by the presence of the perfectly conducting spherical magnetopause, as required by the boundary condition imposed in Sect. 2.1. Equations (7) and (8) imply that the non-radial components of magnetic induction just inside the magnetopause $\left(r=R_{\mathrm{M}}\right)$ are increased by the factor $\{1+[(n+1) / n]\}$ relative to their corresponding values in the absence of the perfectly conducting spherical magnetopause. This general result holds for each of the special cases considered separately in Sects. 3, 4.2 and 4.3 , namely $m=0, m=n$ and $m=n-1$, respectively.

For simplicity, the tilde and the second subscript $(n)$ are omitted in all subsequent equations (apart from those presented in Appendix A).

\section{Zonal magnetic fields}

Consider first the case in which an individual zonal $(m=0)$, or axisymmetric, magnetic multiple of arbitrary degree $n$ is confined by such an ideal spherical magnetopause. It then follows from Eq. (5) that the scalar potential $\left(V_{n}^{0}\right)$ of this individual axisymmetric magnetic multipole of degree $n$ is given by

$$
\begin{aligned}
V_{n}^{0}= & g_{n}^{0} R_{\mathrm{E}}\left(R_{\mathrm{E}} / r\right)^{n+1} \times\left\{1+[(n+1) / n]\left(r / R_{\mathrm{M}}\right)^{2 n+1}\right\} \\
& \times P_{n}^{0}(\cos \theta) .
\end{aligned}
$$

The magnetic field (B) can be found from the equation $\mathbf{B}=-\operatorname{grad}\left(V_{n_{1}}^{0}\right)$. Therefore, since $\mathrm{d} P_{n}^{0}(\cos \theta) / \mathrm{d} \theta=$ $-[n(n+1) / 2]^{1} / \dot{2} P_{n}^{1}(\cos \theta)($ Chapman and Bartels, 1940, Chapter XVII, Eq. 55), the components of the axisymmetric $\left(B_{\phi} \equiv 0\right)$ magnetic field $\mathbf{B}=\left(B_{r}, B_{\theta}\right)$ are given by

$B_{r}=(n+1) g_{n}^{0}\left(R_{\mathrm{E}} / r\right)^{n+2}\left[1-\left(r / R_{\mathrm{M}}\right)^{2 n+1}\right] P_{n}^{0}(\cos \theta)$

and

$$
\begin{aligned}
B_{\theta}= & {[n(n+1) / 2]^{1 / 2} g_{n}^{0}\left(R_{\mathrm{E}} / r\right)^{n+2} } \\
& \times\left\{1+[(n+1) / n]\left(r / R_{\mathrm{M}}\right)^{2 n+1}\right\} P_{n}^{1}(\cos \theta) .
\end{aligned}
$$

These last two equations can also be derived directly from Eqs. (6) and (7), using Eqs. (7) and (8) in the paper by Willis and Young (1987).

The differential equation for the field lines of an axisymmetric magnetic field is $\mathrm{d} r / B_{r}=r \mathrm{~d} \theta / B_{\theta}$,

if the axis of magnetic symmetry is assumed to coincide with the polar axis of the system of spherical polar coordinates defined in Sect. 2. This last assumption involves no real loss of generality. Substituting Eqs. (10) and (11) into Eq. (12) yields the following differential equation for the magnetic field lines inside the axisymmetric spherical magnetosphere

$$
\begin{gathered}
\frac{\left\{1+[(n+1) / n]\left(r / R_{\mathrm{M}}\right)^{2 n+1}\right\}}{r\left[1-\left(r / R_{\mathrm{M}}\right)^{2 n+1}\right]} \mathrm{d} r \\
=\left[\frac{2(n+1)}{n}\right]^{1 / 2} \frac{P_{n}^{0}(\cos \theta)}{P_{n}^{1}(\cos \theta)} \mathrm{d} \theta .
\end{gathered}
$$

The right-hand side of this last equation is identical to the right-hand side of Eq. (11) in the paper by Willis and Young (1987) and can be integrated by the method described in that paper (see also Jeffreys, 1988). Moreover, the left-hand side of Eq. (13) can be expressed as the sum of the two terms $r^{-1} \mathrm{~d} r$ and $[(2 n+1) / n]\left(r^{2 n} / R_{\mathrm{M}}^{2 n+1}\right)\left[1-\left(r / R_{\mathrm{M}}\right)^{2 n+1}\right]^{-1} \mathrm{~d} r$, both of which can be integrated immediately. Using this approach, it is found that Eq. (13) can be integrated to give

$r=r_{n}\left\{\left[1-\left(r / R_{\mathrm{M}}\right)^{2 n+1}\right]\left[\left|\sin \theta P_{n}^{1}(\cos \theta)\right|\right]\right\}^{1 / n}$,

where $r_{n}$ denotes a constant of integration. As in the paper by Willis and Young (1987), the parameter $r_{n}$ specifies a particular axisymmetric shell of field lines; each field line on the shell lies in a meridian plane $(\phi=$ constant). The generalization of Eq. (14) to the case of an arbitrary linear combination of axisymmetric multipoles is presented in Appendix B in terms of an analogy with the solution of an equivalent problem in hydrodynamics (Lamb, 1945).

The interpretation of the parameter $r_{n}$ in Eq. (14) involves a subtlety that warrants explanation. The term inside the $\{$ curly $\}$ brackets on the right-hand side of this equation becomes infinitesimally small as $r \rightarrow R_{\mathrm{M}}$ and hence this equation is meaningful mathematically only if $r_{n} \rightarrow \infty$ as $r \rightarrow R_{\mathrm{M}}$. Therefore, the field lines that lie on the ideal spherical magnetopause surface are those for which the parameter $r_{n}$ is infinitely large. This conclusion may be understood physically by considering the simple case of a magnetic dipole $(n=1)$. In the absence of a confining spherical magnetopause $\left(R_{\mathrm{M}} \rightarrow \infty\right)$, Eq. (14) reduces to the well-known equation for dipolar field lines, namely

$r=r_{1} \sin ^{2} \theta$,

where the parameter $r_{1}$ specifies the geocentric distance at which an individual field line (or shell of field lines) crosses the equatorial plane $(\theta=\pi / 2)$. Imagine a perfectly conducting spherical magnetopause that contracts radially in such a way that its radius decreases from an initial infinitely large value to the final finite value $R_{\mathrm{M}}$. During its inward radial motion, this perfectly conducting spherical magnetopause sweeps up all the magnetic flux initially distributed throughout the vol- 
ume $R_{\mathrm{M}}<r<\infty$ (the "snowplough" effect) and redistributes this flux within the spherical magnetospheric cavity $\left(r \leq R_{\mathrm{M}}\right)$. It is then clear topologically that the field lines on the spherical magnetopause are equivalent to those that extend initially to an infinitely large distance from the dipole (i.e. $r_{1}=\infty$ ), namely those that lie arbitrarily close to the dipole axis $(\theta=0, \pi)$ at the surface of the Earth.

An analogous physical argument applies to the magnetic field lines of the individual axisymmetric multipole of degree $n$, which are identified by particular values of the parameter $r_{n}$ in Eq. (14). The field lines that lie on the spherical magnetopause are those that would extend to an infinitely large distance from the multipole (i.e. $r_{n}=\infty$ ) in the absence of the confining magnetopause. This physical explanation can be confirmed conceptually by judicious inspection of Fig. 2, which is presented and discussed in Sect. 6.1, and by comparison of this figure with Fig. 1 in Willis and Young (1987).

\section{Sectorial magnetic fields}

\subsection{The magnetic scalar potentials}

In this section exact equations are derived for the field lines of two special non-axisymmetric magnetic multipoles, each of which is assumed to be confined by the ideal, perfectly conducting, spherical magnetopause defined in Sects. 1 and 2. Following the work of Willis and Gardiner (1988), these two special magnetic multipoles have arbitrary degree $n$ but restricted order $m$, namely (1) $m=n$ and (2) $m=n-1$. Thus this study extends the earlier work to the case in which the field lines of these special non-axisymmetric magnetic multipoles are constrained to lie within the idealized spherical magnetosphere.

As noted in the earlier investigation (Willis and Gardiner, 1988), Schmidt's partially normalized form of the associated Legendre function $P_{n}^{m}(\cos \theta)$ can be expressed as a finite series as follows if $m>0$ (Chapman and Bartels, 1940, Chapter XVII, Eqs. 10 and 20):

$$
\begin{aligned}
P_{n}^{m}(\cos \theta)= & \frac{(2 n) !}{2^{n} \cdot n !}\left[\frac{2}{(n+m) !(n-m) !}\right]^{1 / 2} \sin ^{m} \theta \\
& \times\left[\cos ^{n-m} \theta-\frac{(n-m)(n-m-1)}{2(2 n-1)} \cos ^{n-m-2} \theta\right. \\
& +\frac{(n-m)(n-m-1)(n-m-2)(n-m-3)}{2 \cdot 4(2 n-1)(2 n-3)} \\
& \left.\times \cos ^{n-m-4} \theta-\cdots \cdots \cdots\right] .
\end{aligned}
$$

In the degenerate case $m=0$, Eq. (16) yields a value of $P_{n}^{0}(\cos \theta)$ that is too large by a factor $2^{1 / 2}$ (see Chapman and Bartels, 1940, Chapter XVII, Eqs.19 and 20). However, in the present context, this degenerate case arises only for a magnetic dipole $(m=0$, $n=1)$ aligned with the polar axis $(\theta=0, \pi)$ and even in this situation the use of Eq. (16) gives the correct configuration of magnetic field lines because the configuration is independent of the magnitude of the scaling factor.

For the two individual non-axisymmetric magnetic multipoles considered here, namely symmetric sectorial multipoles $(m=n)$ and antisymmetric sectorial multipoles $(m=n-1)$, it is clear from Eqs. (5) and (16) that the corresponding scalar potentials become, respectively,

$$
\begin{aligned}
V_{n}^{n}= & \frac{[2 \cdot(2 n) !]^{1 / 2}}{2^{n} \cdot n !} c_{n}^{n} R_{\mathrm{E}}\left(R_{\mathrm{E}} / r\right)^{n+1} \\
& \times\left\{1+[(n+1) / n]\left(r / R_{\mathrm{M}}\right)^{2 n+1}\right\} \\
& \times \sin ^{n} \theta \cos \left[n\left(\phi-\phi_{n}^{n}\right)\right]
\end{aligned}
$$

and

$$
\begin{aligned}
V_{n}^{n-1}= & \frac{(2 n) !}{2^{n} \cdot n !}\left[\frac{2}{(2 n-1) !}\right]^{1 / 2} c_{n}^{n-1} R_{\mathrm{E}}\left(R_{\mathrm{E}} / r\right)^{n+1} \\
& \times\left\{1+[(n+1) / n]\left(r / R_{\mathrm{M}}\right)^{2 n+1}\right\} \\
& \times \sin ^{n-1} \theta \cos \theta \cos \left[(n-1)\left(\phi-\phi_{n}^{n-1}\right)\right],
\end{aligned}
$$

where, in general $c_{n}^{m}=\left|\left[\left(g_{n}^{m}\right)^{2}+\left(h_{n}^{m}\right)^{2}\right]^{1 / 2}\right|$ and $\phi_{n}^{m}=(1 / m) \arctan \left(h_{n}^{m} / g_{n}^{m}\right)$ for $0 \leq m \leq n\left(c_{n}^{0} \equiv g_{n}^{0}\right.$, $\left.h_{n}^{0} \equiv 0\right)$ (Roederer, 1972; Willis and Gardiner, 1988). The order $m$ satisfies the condition $m=n$ in Eq. (17) and the condition $m=n-1$ in Eq. (18).

\subsection{Symmetric sectorial multipoles $(m=n)$}

The magnetic-field components of a symmetric sectorial multipole $(m=n)$, which is confined within the idealized spherical magnetosphere, can be found from the equation $\mathbf{B}=-\operatorname{grad}\left(V_{n}^{n}\right)$, in which $V_{n}^{n}$ is defined by Eq. (17); they are

$$
\begin{aligned}
B_{r}= & (n+1) C_{n}^{n} r^{-(n+2)}\left\{1-\left(r / R_{\mathrm{M}}\right)^{2 n+1}\right\} \\
& \times \sin ^{n} \theta \cos \left[n\left(\phi-\phi_{n}^{n}\right)\right], \\
B_{\theta}= & -n C_{n}^{n} r^{-(n+2)}\left\{1+[(n+1) / n]\left(r / R_{\mathrm{M}}\right)^{2 n+1}\right\} \\
& \times \sin ^{n-1} \theta \cos \theta \cos \left[n\left(\phi-\phi_{n}^{n}\right)\right], \\
B_{\phi}= & n C_{n}^{n} r^{-(n+2)}\left\{1+[(n+1) / n]\left(r / R_{\mathrm{M}}\right)^{2 n+1}\right\} \\
& \times \sin ^{n-1} \theta \sin \left[n\left(\phi-\phi_{n}^{n}\right)\right],
\end{aligned}
$$

where

$C_{n}^{n}=\frac{[2 \cdot(2 n) !]^{1 / 2}}{2^{n} \cdot n !} c_{n}^{n} R_{\mathrm{E}}^{n+2}$.

Referred to a system of spherical polar coordinates, the set of differential equations for the field lines of a general magnetic field is of the form

$\mathrm{d} r / B_{r}=r \mathrm{~d} \theta / B_{\theta}=r \sin \theta \mathrm{d} \phi / B_{\phi}$.

Substituting Eqs. (19), (20) and (21) into (23) yields the following three differential equations for the magnetic field lines of an individual symmetric sectorial magnetic multipole $(m=n)$ confined within the spherical magnetosphere of radius $R_{\mathrm{M}}$ : 


$$
\frac{\left\{1+[(n+1) / n]\left(r / R_{\mathrm{M}}\right)^{2 n+1}\right\} \mathrm{d} r}{r\left[1-\left(r / R_{\mathrm{M}}\right)^{2 n+1}\right]}=-\frac{(n+1)}{n} \tan \theta \mathrm{d} \theta,
$$

$$
\begin{aligned}
& \frac{\sec ^{2} \theta \mathrm{d} \theta}{\tan \theta}=-\cot \left[n\left(\phi-\phi_{n}^{n}\right)\right] \mathrm{d} \phi, \\
& \frac{\left\{1+[(n+1) / n]\left(r / R_{\mathrm{M}}\right)^{2 n+1}\right\} \mathrm{d} r}{r\left[1-\left(r / R_{\mathrm{M}}\right)^{2 n+1}\right]} \\
& \quad=\frac{(n+1)}{n} \sin ^{2} \theta \cot \left[n\left(\phi-\phi_{n}^{n}\right)\right] \mathrm{d} \phi .
\end{aligned}
$$

It follows from the functional form of Eqs. (19), (20) and (21) that the three differential Eqs. (24), (25) and (26) are valid if $0<\theta<\pi$. The polar axis $(\theta=0, \pi)$ is a magnetic neutral line if $n>1$ and no singularities exist on this axis in the special case $n=1$, which corresponds to a dipole with its axis lying in the equatorial plane (Roederer, 1972). However, it should be noted that Eq. (26) can be derived directly from Eqs. (24) and (25), which implies that the magnetic field lines are defined uniquely by the latter two (independent) equations. Nevertheless, Eq. (26) is still of value because it defines those field lines that lie in the equatorial plane $(\theta=\pi / 2)$.

Equations (24) and (25) may be integrated immediately to give

$r=r_{n}^{n}\left\{\left[1-\left(r / R_{\mathrm{M}}\right)^{2 n+1}\right](|\cos \theta|)^{n+1}\right\}^{1 / n}$

and

$\left|\tan ^{n} \theta \sin \left[n\left(\phi-\phi_{n}^{n}\right)\right]\right|=K_{n}^{n}$,

where $r_{n}^{n}$ and $K_{n}^{n}$ denote constants of integration. It follows from Eq. (20) that the magnetic field of a symmetric sectorial multipole confined within the spherical magnetosphere of radius $R_{\mathrm{M}}$ is locally parallel to the equatorial plane (i.e. $B_{\theta}=0$ if $\theta=\pi / 2$ ). For a field line lying in the equatorial plane $(\theta=\pi / 2)$, Eq. (26) may also be integrated at once to give

$$
\begin{aligned}
r= & \left(r_{n}^{n}\right)^{\prime}\left\{\left[1-\left(r / R_{\mathrm{M}}\right)^{2 n+1}\right]\right. \\
& \left.\times\left\{\left|\sin \left[n\left(\phi-\phi_{n}^{n}\right)\right]\right|\right\}^{(n+1) / n}\right\}^{1 / n},
\end{aligned}
$$

where $\left(r_{n}^{n}\right)^{\prime}$ denotes another constant of integration.

\subsection{Antisymmetric sectorial multipoles $(m=n-1)$}

The magnetic-field components of an antisymmetric sectorial multipole $(m=n-1)$, which is confined within the idealized spherical magnetosphere, can be found from the equation $\mathbf{B}=-\operatorname{grad}\left(V_{n}^{n-1}\right)$, in which $V_{n}^{n-1}$ is defined by Eq. (18); they are

$$
\begin{aligned}
B_{r}= & (n+1) C_{n}^{n-1} r^{-(n+2)}\left[1-\left(r / R_{\mathrm{M}}\right)^{2 n+1}\right] \\
& \times \sin ^{n-1} \theta \cos \theta \cos \left[(n-1)\left(\phi-\phi_{n}^{n-1}\right)\right], \\
B_{\theta}= & -C_{n}^{n-1} r^{-(n+2)}\left\{1+[(n+1) / n]\left(r / R_{\mathrm{M}}\right)^{2 n+1}\right\} \\
& \times\left[(n-1) \cos ^{2} \theta-\sin ^{2} \theta\right] \sin ^{n-2} \theta \\
& \times \cos \left[(n-1)\left(\phi-\phi_{n}^{n-1}\right)\right],
\end{aligned}
$$

$$
\begin{aligned}
B_{\phi}= & (n-1) C_{n}^{n-1} r^{-(n+2)}\left\{1+[(n+1) / n]\left(r / R_{\mathrm{M}}\right)^{2 n+1}\right\} \\
& \times \sin ^{n-2} \theta \cos \theta \sin \left[(n-1)\left(\phi-\phi_{n}^{n-1}\right)\right],
\end{aligned}
$$

where

$$
C_{n}^{n-1}=\frac{(2 n) !}{2^{n} \cdot n !}\left[\frac{2}{(2 n-1) !}\right]^{1 / 2} c_{n}^{n-1} R_{\mathrm{E}}^{n+2} .
$$

As in the case of a symmetric sectorial multipole, substituting, Eqs. (30), (31) and (32) into Eq. (23) yields the following three differential equations for the magnetic field lines of an individual antisymmetric sectorial multipole $(m=n-1)$ confined within the spherical magnetosphere of radius $R_{\mathrm{M}}$ :

$$
\begin{gathered}
\frac{\left\{1+[(n+1) / n]\left(r / R_{\mathrm{M}}\right)^{2 n+1}\right\} \mathrm{d} r}{r\left[1-\left(r / R_{\mathrm{M}}\right)^{2 n+1}\right]} \\
=\frac{-(n+1) \sin \theta \cos \theta \mathrm{d} \theta}{\left[(n-1) \cos ^{2} \theta-\sin ^{2} \theta\right]}, \\
\frac{\cos \theta \mathrm{d} \theta}{\sin \theta\left[(n-1) \cos ^{2} \theta-\sin ^{2} \theta\right]} \\
=\frac{-\cot \left[(n-1)\left(\phi-\phi_{n}^{n-1}\right)\right] \mathrm{d} \phi}{(n-1)},
\end{gathered}
$$

and

$$
\begin{aligned}
\frac{\left\{1+[(n+1) / n]\left(r / R_{\mathrm{M}}\right)^{2 n+1}\right\} \mathrm{d} r}{r\left[1-\left(r / R_{\mathrm{M}}\right)^{2 n+1}\right]} & \\
= & \frac{(n+1) \sin ^{2} \theta \cot \left[(n-1)\left(\phi-\phi_{n}^{n-1}\right)\right] \mathrm{d} \phi}{(n-1)} .
\end{aligned}
$$

It is clear from the functional form of Eqs. (30), (31) and (32) that the three differential Eqs. (34), (35) and (36) are valid if either $0<\theta<\pi / 2$ or $\pi / 2<\theta<\pi$. The polar axis $(\theta=0, \pi)$ is a magnetic neutral line if $n>2$ and no singularities exist on this axis in the degenerate case $n=1$ and the special case $n=2$. The degenerate case $n=1$ corresponds to a zonal (or axisymmetric) dipole, which is considered in greater detail in Sect. 3, and the special case $n=2$ corresponds to a "normal" (or planar) quadrupole aligned with the polar axis (Roederer, 1972). As in the case $m=n$, Eq. (36) can be derived from Eqs. (34) and (35), which implies that the field lines are defined uniquely by the latter two (independent) equations. Moreover, the magnetic field is locally perpendicular to the equatorial plane $(\theta=\pi / 2)$ in the case of an antisymmetric sectorial magnetic multipole $(m=n-1)$ and therefore Eq. (36) provides no useful additional information.

Equation (34) can be integrated immediately by standard methods to give (Beyer, 1984; integral 346)

$$
\begin{aligned}
r= & r_{n}^{n-1}\left\{\left[1-\left(r / R_{\mathrm{M}}\right)^{2 n+1}\right]\right. \\
& \left.\times\left[\left|(n-1) \cos ^{2} \theta-\sin ^{2} \theta\right|\right]^{(n+1) / 2}\right\}^{1 / n},
\end{aligned}
$$

where $r_{n}^{n-1}$ is a constant of integration. If $n=1(m=0)$, Eq. (37) simplifies to the form

$r=r_{1}^{0}\left[1-\left(r / R_{\mathrm{M}}\right)^{3}\right] \sin ^{2} \theta$. 
Since $P_{1}^{1}(\cos \theta)=\sin \theta$, this last equation can also be obtained by putting $n=1$ in Eq. (14), which is the general equation for the field lines of an individual axisymmetric multipole of degree $n$, aligned with the polar axis $(\theta=0, \pi)$ and confined by the perfectly conducting spherical magnetopause of radius $R_{\mathrm{M}}$. If $n=1(m=0)$, the (axisymmetric) magnetic field lines are confined to geographic meridian planes $\left(B_{\phi} \equiv 0\right)$, as implied by Eqs. (30), (31) and (32). In this degenerate case Eq. (35) is nullified. However, if $n>1$, Eq. (35) can be rewritten in a more convenient form (Willis and Gardiner, 1988), which can be integrated immediately by standard techniques to give (Beyer, 1984; integral 346)

$\frac{\left|\sin ^{n-1} \theta \sin \left[(n-1)\left(\phi-\phi_{n}^{n-1}\right)\right]\right|}{\left[\left|(n-1) \cos ^{2} \theta-\sin ^{2} \theta\right|\right]^{(n-1) / 2}}=K_{n}^{n-1}$,

where $K_{n}^{n-1}$ is another constant of integration.

\section{General properties of the magnetic field lines}

The purpose of this section is to derive some general geometrical properties of the magnetic field lines for the three special cases of an individual magnetic multipole confined by a perfectly conducting (concentric) spherical magnetopause, namely: (1) zonal multipoles $(m=0)$; (2) symmetric sectorial multipoles $(m=n)$; and (3) antisymmetric sectorial multipoles $(m=n-1)$.

\subsection{Zonal multipoles $(m=0)$}

It follows from Eqs. (10) and (11) that the magnetic field of the confined zonal multipole of degree $n$ is horizontal $\left(B_{r}=0\right)$ at values of $\theta$ that satisfy the equation $P_{n}^{0}(\cos \theta)=0$ and also everywhere on the spherical magnetopause $\left(r=R_{\mathrm{M}}\right)$. Likewise, the magnetic field is vertical $\left(B_{\theta}=0\right)$ at values of $\theta$ that satisfy the equation $P_{n}^{1}(\cos \theta)=0$. The numerical values of $\theta$ that satisfy the equations $P_{n}^{0}(\cos \theta)=0$ and $P_{n}^{1}(\cos \theta)=0$ have been tabulated by Chapman and Bartels (1940, Table D) for $1 \leq n \leq 7$.

It is immediately obvious from Eq. (14) that $r$ attains its minimum value, $r=0$, if either $\sin \theta=0$ or $P_{n}^{1}(\cos \theta)=0$, provided $r_{n}$ is finite. (As noted in Sect. 3, $r_{n} \rightarrow \infty$ as $r \rightarrow R_{\mathrm{M}}$.) In fact, $\sin \theta$ is a factor of $P_{n}^{1}(\cos \theta)$ and hence $\theta=0$ and $\theta=\pi$ are automatically roots of the equation $P_{n}^{1}(\cos \theta)=0$ (Chapman and Bartels, 1940, Chapter XVII, Eqs. 10 and 20; Matsushita and Campbell, 1967, Volume II, Appendix 3). Therefore, the magnetic field is vertical (radial) everywhere on the axis of magnetic symmetry $(\theta=0, \pi)$ and on the set of cones $\theta=\theta_{i}$ where $\theta_{i}(1 \leq i \leq n-1)$ denotes one of the $(n-1)$ roots of the equation $P_{n}^{1}(\cos \theta)=0$ in the range $0<\theta<\pi$ (Chapman and Bartels, 1940, Chapter XVII, Sect. 17.6). If $\theta_{i}=\pi / 2$ for a particular value of $i$, which occurs if $n$ is even (i.e. if $n-1$ is odd), the corresponding cone $\theta=\theta_{i}$ degenerates to the equatorial plane. Hence the magnetic field lines are straight and vertical on the axis of magnetic symmetry and on the cones $\theta=\theta_{i}$. Every other field line is curved and becomes vertical (radial) only asymptotically at the origin. All these results are exactly the same as those in the absence of the perfectly conducting spherical magnetopause.

An important new result arises as a direct consequence of the presence of the perfectly conducting spherical magnetopause. Since the radial component of the magnetic field vanishes everywhere on this surface by definition, neutral points, or rings, occur wherever a radial magnetic field line in the magnetosphere intersects the spherical magnetopause. Therefore, two neutral points exist where the axis of magnetic symmetry $(\theta=0, \pi)$ intersects the spherical magnetopause $\left(r=R_{\mathrm{M}}\right)$. Likewise, $(n-1)$ neutral rings exist where the cones $\theta=\theta_{i}$ intersect this same surface, where $\theta_{i}(1 \leq i \leq n-1)$ again denotes one of the $(n-1)$ roots of the equation $P_{n}^{1}(\cos \theta)=0$ in the range $0<\theta<\pi$.

Similarly, by an argument analogous to that presented by Willis and Young (1987, see Sect. 6), it can be shown that the values of $\theta$ at which suites of similar magnetic field lines reach their maximum radial distance from the origin, $r_{m}$, are given by the $n$ roots of the equation $P_{n}^{0}(\cos \theta)=0$. This is the condition for the magnetic field to be horizontal. Stated alternatively, the magnetic field is horizontal everywhere on the set of cones $\theta=\theta_{i}$, where $\theta_{i}(1 \leq i \leq n)$ now denotes one of the $n$ roots of the equation $P_{n}^{0}(\cos \theta)=0$ in the range $0<\theta<\pi$ (Chapman and Bartels 1940, Chapter XVII, Sect. 17.6). Therefore, the values of $\theta$ at which suites of similar magnetic field lines reach their maximum radial distance from the origin are unchanged by the presence of the perfectly conducting spherical magnetopause, although the maximum radial distances actually achieved obviously do depend on the magnitude of the radius of the magnetopause $\left(R_{\mathrm{M}}\right)$.

Equation (14) provides a complete description of the magnetic field lines for an individual zonal (axisymmetric) magnetic multipole $(m=0)$ confined by a perfectly conducting (concentric) spherical magnetopause. This equation defines, for different values of $r_{n}$, a set of surfaces of revolution on which families of magnetic field lines lie. As the magnetic field is axisymmetric, every field line is confined to a magnetic meridian plane. The actual configurations of the magnetic field lines for representative low-degree zonal multipoles $(m=0)$ are presented and discussed in Sect. 6.1.

\subsection{Symmetric sectorial multipoles $(m=n)$}

It follows from Eqs. (19), (20) and (21) that the magnetic field of the confined symmetric sectorial multipole of degree $n$ is locally parallel to the equatorial plane; that is $B_{\theta}=0$ if $\theta=\pi / 2$. Similarly, the magnetic field is locally perpendicular to the $2 n$ meridional planes $\phi=\phi_{n}^{n}+(2 k+1) \pi / 2 n$, where $k=0,1,2, \ldots, 2 n-1$; that is $B_{r}=B_{\theta}=0, B_{\phi} \neq 0$ everywhere in these planes apart from the polar axis $(\theta=0, \pi)$, which is a neutral line $\left(B_{r}=B_{\theta}=B_{\phi}=0\right)$ if $n>1$. The special case $m=n=1$ corresponds to a magnetic dipole lying in 
the equatorial plane (Roederer, 1972). The magnetic field is purely meridional $\left(B_{\phi}=0\right)$ in the $2 n$ planes $\phi=\phi_{n}^{n}+k \pi / n(k=0,1,2, \ldots, 2 n-1)$ and becomes purely radial $\left(B_{\theta}=B_{\phi}=0\right)$ at the intersections of these meridional planes with the equatorial plane $(\theta=\pi / 2)$. Magnetic field lines that are confined to the special meridional planes $\left(B_{\phi}=0\right)$ or the equatorial plane $\left(B_{\theta}=0\right)$, when the symmetric sectorial multipole is in free space, continue to be confined to these same planes when the perfectly conducting spherical magnetopause is introduced.

As a result of the presence of this perfectly conducting surface, however, neutral points occur wherever a radial field line in the magnetosphere intersects the spherical magnetopause. Therefore, $2 n$ neutral points exist at the intersections of the $2 n$ meridional planes $\phi=\phi_{n}^{n}+k \pi / n(k=0,1,2, \ldots, 2 n-1)$ with both the equatorial plane $(\theta=\pi / 2)$ and the spherical magnetopause $\left(r=R_{\mathrm{M}}\right)$. In addition, the polar axis $(\theta=0, \pi)$ is a neutral line if $n>1$ and thus neutral points inevitably exist on the spherical magnetopause surface at the two poles.

The three Eqs. (27), (28) and (29) provide a complete description of the magnetic field lines for the general symmetric sectorial magnetic multipole $(m=n)$ confined by a perfectly conducting (concentric) spherical magnetopause. Equation (27) defines, for different values of $r_{n}^{n}$, a set of surfaces of revolution on which families of magnetic field lines lie. As noted previously, the magnetic field is purely meridional $\left(B_{\phi}=0\right)$ in the $2 n$ planes $\phi=\phi_{n}^{n}+k \pi / n(k=0,1,2 \ldots, 2 n-1)$; therefore, the surfaces of revolution define the planar field lines in these $2 n$ planes. Equation (28) defines, for different values of $K_{n}^{n}$, the magnetic meridian curves on a sphere or, alternatively, the singly curved surfaces whose intersections with the surfaces of revolution defined by Eq. (27) are the general field lines of the confined symmetric sectorial magnetic multipole $(m=n)$. Since Eq. (28) is identical to Eq. (23) in the paper by Willis and Gardiner (1988), the magnetic meridian curves on a sphere are unchanged by the confinement of the symmetric sectorial multipole $(m=n)$ by a perfectly conducting spherical magnetopause. Finally Eq. (29) defines, for various values of $\left(r_{n}^{n}\right)^{\prime}$, the set of magnetic field lines that lie entirely in the equatorial plane. The actual configurations of the magnetic field lines for representative low-degree symmetric sectorial multipoles $(m=n)$ are presented and discussed in Sect. 6.2.

\subsection{Antisymmetric sectorial multipoles $(m=n-1)$}

It follows from Eqs. (30), (31) and (32) that there are $2(n-1)$ neutral lines $\left(B_{r}=B_{\theta}=B_{\phi}=0\right)$ in the equatorial plane $(\theta=\pi / 2)$ of a confined antisymmetric sectorial multipole; these are located at the intersections of the equator with the $2(n-1)$ meridional planes $\phi=\phi_{n}^{n-1}+(2 k+1) \pi / 2(n-1)$, where $k=0,1,2$, $\ldots, 2 n-3$. Elsewhere, the magnetic field is locally perpendicular to the equatorial plane; that is, $B_{r}=$ $B_{\phi}=0, B_{\theta} \neq 0$ if $\theta=\pi / 2$. Moreover, the magnetic field is locally perpendicular to the $2(n-1)$ meridional planes $\phi=\phi_{n}^{n-1}+(2 k+1) \pi / 2(n-1)$; that is, $B_{r}=$ $B_{\theta}=0, B_{\phi} \neq 0$ everywhere in these planes apart from the polar axis, which is a neutral line if $n>2$, and the $2(n-1)$ equatorial neutral lines. The degenerate case $m=0, n=1$ corresponds to a zonal dipole coincident with the polar axis and the special case $m=1, n=2$ corresponds to a "normal" (or planar) quadrupole aligned with the polar axis (Roederer, 1972). Finally, the magnetic field is purely meridional $\left(B_{\phi}=0\right)$ in the $2(n-1) \quad$ planes $\phi=\phi_{n}^{n-1}+k \pi /(n-1) \quad(k=0,1,2$, $\ldots, 2 n-3)$ and becomes purely radial $\left(B_{\theta}=B_{\phi}=0\right)$ at the intersections of these meridional planes with the circular conical surface $\theta=\arctan \left[(n-1)^{1 / 2}\right], \quad \theta=$ $\pi-\arctan \left[(n-1)^{1 / 2}\right]$. Magnetic field lines that are confined to the special meridional planes $\left(B_{\phi}=0\right)$, when the antisymmetric sectorial multipole is in free space, continue to be confined to these same planes when the perfectly conducting spherical magnetopause is introduced.

As a result of the presence of this perfectly conducting surface, however, neutral points occur wherever a radial field line in the magnetosphere intersects the spherical magnetopause. In the degenerate case $m=0$, $n=1$, two neutral points exist where the polar axis $(\theta, \pi)$ intersects the spherical magnetopause $\left(r=R_{\mathrm{M}}\right)$ : in this degenerate case the polar axis is also the axis of magnetic symmetry. In the general case, $4(n-1)$ neutral points exist at the intersections of the $2(n-1)$ meridional planes $\phi=\phi_{n}^{n-1}+k \pi /(n-1) \quad(k=0,1,2, \ldots$, $2 n-3)$ with the circular conical surface $\theta=$ $\arctan \left[(n-1)^{1 / 2}\right], \theta=\pi-\arctan \left[(n-1)^{1 / 2}\right]$; in this case there are $2(n-1)$ neutral points in each hemisphere. As already noted, the polar axis $(\theta=0, \pi)$ is a neutral line if $n>2$ and there are also $2(n-1)$ equatorial neutral lines at the intersections of the equator $(\theta=\pi / 2)$ with the $2(n-1)$ planes $\phi=\phi_{n}^{n-1}+(2 k+1) \pi / 2(n-1)$, where $k=0,1,2, \ldots, 2 n-3$. Therefore, neutral points inevitably exist where the polar axis and the $2(n-1)$ equatorial neutral lines intersect the spherical magnetopause.

The two Eqs. (37) and (39) provide a complete description of the magnetic field lines for the special tesseral (or "general antisymmetric sectorial") magnetic multipole specified by $m=n-1$. Equation (37) defines, for different values of $r_{n}^{n-1}$, a set of surfaces of revolution on which families of magnetic field lines lie. As noted previously, the magnetic field is purely meridional $\left(B_{\phi}=0\right)$ in the $2(n-1)$ planes $\phi=\phi_{n}^{n-1}+k \pi /(n-1)$; therefore, the surfaces of revolution define the planar field lines in these $2(n-1)$ meridional planes. Equation (39) defines, for different values of $K_{n}^{n-1}$, the magnetic meridian curves on a sphere or, alternatively, the singly curved surfaces whose intersections with the surfaces of revolution defined by Eq. (37) are the general field lines of the special tesseral magnetic multipole $(m=n-1)$. Since Eq. (39) is identical to Eq. (29) in the paper by Willis and Gardiner (1988), the magnetic meridian curves on a sphere are unchanged by the confinement of the special tesseral magnetic multipole $(m=n-1)$ by a perfectly conducting spherical magnetopause. The actual configurations of the magnetic field lines for 
representative low-degree antisymmetric sectorial multipoles $(m=n-1)$ are presented and discussed in Sect. 6.3.

\section{Configurations of the magnetic field lines in the outer magnetosphere}

The purpose of this section is to illustrate and discuss the characteristic configurations of the magnetic field lines in the outer magnetosphere for the three special cases: (1) zonal multipoles $(m=0)$; (2) symmetric sectorial multipoles $(m=n)$; and (3) antisymmetric sectorial multipoles $(m=n-1)$.

\subsection{Zonal multipoles $(m=0)$}

Figure $2 \mathrm{a}-\mathrm{d}$ shows the magnetic-field-line configurations for the first four low-degree axisymmetric $(m=0)$, or zonal, multipoles, respectively; namely, an axial dipole $(n=1)$, an axial quadrupole $(n=2)$, an axial octupole $(n=3)$ and an axial sedecimupole $(n=4)$. (Some authors use the term "hexadecapole" rather than "sedecimupole"; the latter term was introduced by Winch, 1967, whereas Willis and Young, 1987, used
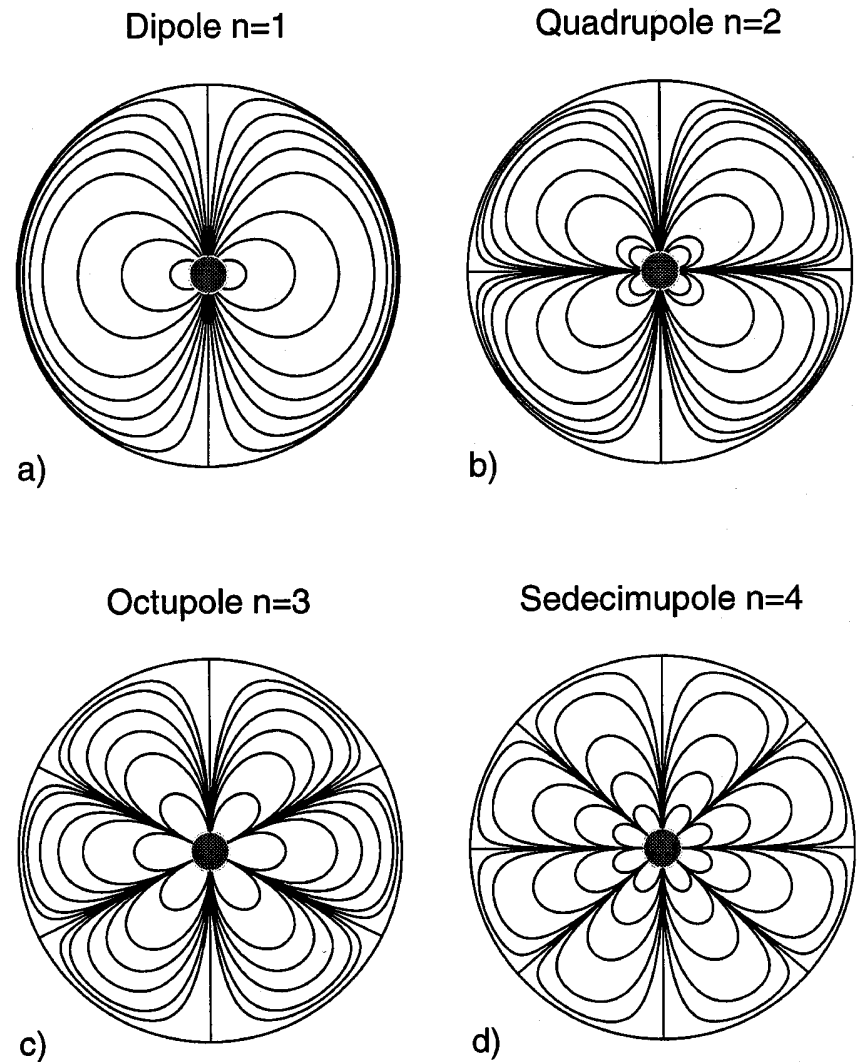

Fig. 2a-d. Illustrative magnetic-field-line configurations for lowdegree zonal (axisymmetric) magnetic multipoles $(m=0)$ : a dipole $(n=1)$, b quadrupole $(n=2)$, c octupole $(n=3)$, and $\mathbf{d}$ sedecimupole $(n=4)$. In this illustrative figure (and in Figs. 3 and 4 ), the radius of the magnetopause is taken to be ten times the radius of the Earth $\left(R_{\mathrm{M}}=10 R_{\mathrm{E}}\right)$ the former term.) These four magnetic-field-line configurations have been calculated from Eq. (14) by choosing an appropriate set of values $\left\{\left(r_{n}\right)_{j}\right\}$ of the parameter $r_{n}$ for each value of $n(1 \leq n \leq 4)$ in the illustrative case for which $R_{\mathrm{M}}=10 R_{\mathrm{E}}$. For each value of $\left(r_{n}\right)_{j}$, the variable $\theta$ is incremented to provide a set of coordinates $\left\{\left(r_{j}, \theta_{j}\right)\right\}$ that specify the individual field line defined by the parameter $\left(r_{n}\right)_{j}$. For finite values of $R_{\mathrm{M}}$, the values of $r_{j}$ are determined from Eq. (14) by finding the appropriate root of this polynomial equation of degree $(2 n+1)$. In the case of a confined zonal magnetic multipole, the four magnetic-field-line configurations presented in Fig. 2a-d show that neutral points exist on the spherical magnetopause surface at the two poles. In addition, $(n-1)$ neutral rings exist on the magnetopause surface and if $n$ is even one of these neutral rings lies in the (magnetic) equatorial plane.

The physical explanation of the compression of a zonal magnetic field may be understood in terms of the reasoning that follows Eq. (14). Since magnetic field lines cannot enter the perfectly diamagnetic surrounding medium, they are confined within the spherical magnetospheric cavity. This confinement is clearly illustrated in Fig. 2a-d, which shows the compression of magnetic field lines by the spherical magnetopause for the first four low-degree axisymmetric multipoles $(1 \leq n \leq 4)$ in the particular case $R_{\mathrm{M}}=10 R_{\mathrm{E}}$. In order to demonstrate more generally how the spherical magnetopause compresses magnetic field lines in the outer magnetosphere, it is instructive to calculate the maximum radial distance, $r_{m}$, of a specified field line for various values of $R_{\mathrm{M}}$. The maximum radial distances achieved by a selection of field lines can then be compared with the corresponding values in the absence of a perfectly conducting spherical magnetopause. The comparisons are shown in Tables $1-4$, in which the left-hand columns give the maximum (non-dimensional) radial distances $\left(r_{n} / R_{\mathrm{E}} ; 1 \leq n \leq 4\right)$ in the absence of a confining magnetopause $\left(R_{\mathrm{M}} \rightarrow \infty\right)$. The remaining columns in these tables give the corresponding maximum (non-dimensional) radial distances $\left(r_{m} / R_{\mathrm{E}}\right)$ for several illustrative values of the cavity radius $\left(R_{\mathrm{M}}\right)$ in the range $10 \leq R_{\mathrm{M}} / R_{\mathrm{E}} \leq 1000$. All distances in these tables are expressed in Earth-radii $\left(R_{\mathrm{E}}\right)$, measured from the centre of the Earth, and are given to five significant figures in order to show small changes. The values of $\theta$ at which magnetic field lines achieve their maximum radial distances are given (to the nearest second of arc) in the captions to Tables $1-4$. The precise positions of the neutral points $(P)$ and neutral rings $(R)$ that lie on the magnetopause surface, as illustrated in Fig. $2 \mathrm{a}-\mathrm{d}$ for the first four zonal magnetic multipoles $(1 \leq n \leq 4)$, are listed in Table 5.

For convenience, Tables 1-4 show the compression of magnetic field lines, measured by the value(s) of the parameter $r_{m} / R_{\mathrm{E}}$ relative to the value of the parameter $r_{n} / R_{\mathrm{E}}(1 \leq n \leq 4)$, rather than the limiting value of the parameter $r_{m} / R_{\mathrm{E}}$ as $R_{\mathrm{M}} / R_{\mathrm{E}} \rightarrow \infty$. The limiting value of the parameter $r_{m} / R_{\mathrm{E}}$ is only equal to the value of the parameter $r_{n} / R_{\mathrm{E}}$ in the simplest case of a magnetic dipole $(n=1)$; hence the use of the terms directly 
Table 1. Maximum radial distances $\left(r_{m} / R_{\mathrm{E}} ; \theta=90^{\circ}\right)$ of selected magnetic field lines, for various values of the radius of the perfectly conducting spherical magnetopause $\left(R_{\mathrm{M}} / R_{\mathrm{E}}\right)$, in the case of an axial dipole $(n=1)$. The value of the parameter $r_{1} / R_{\mathrm{E}}$ specifies directly (see Sect. 6.1) the corresponding maximum (equatorial) radial distance of each selected (dipolar) field line in the absence of the spherical magnetopause $\left(R_{\mathrm{M}} / R_{\mathrm{E}} \rightarrow \infty\right)$. All distances in Tables 1-4 are expressed in Earth-radii $\left(R_{\mathrm{E}}\right)$, measured from the centre of the Earth, and are given to five significant figures

\begin{tabular}{|c|c|c|c|c|c|c|c|}
\hline$r_{1} / R_{\mathrm{E}}$ & \multicolumn{7}{|l|}{$R_{\mathrm{M}} / R_{\mathrm{E}}$} \\
\hline 10 & 6.8233 & 9.4584 & 9.9219 & 9.9900 & 9.9994 & 9.9999 & 10.000 \\
\hline 25 & 8.6756 & 17.058 & 22.670 & 24.627 & 24.975 & 24.997 & 25.000 \\
\hline 50 & 9.3344 & 20.878 & 34.116 & 45.340 & 49.609 & 49.950 & 49.994 \\
\hline 100 & 9.6668 & 22.922 & 41.756 & 68.233 & 94.585 & 99.219 & 99.900 \\
\hline 1000 & 9.9667 & 24.792 & 49.167 & 96.668 & 229.22 & 417.56 & 682.33 \\
\hline $10^{6}$ & 10.000 & 25.000 & 49.999 & 99.997 & 249.98 & 499.92 & 999.67 \\
\hline
\end{tabular}

Table 2. Maximum radial distances $\left(r_{m} / R_{\mathrm{E}} ; \theta=54^{\circ} 44^{\prime} 08^{\prime \prime}, 125^{\circ}\right.$ $15^{\prime} 52^{\prime \prime}$ ) of selected magnetic field lines, for various values of the radius of the perfectly conducting spherical magnetopause $\left(R_{\mathrm{M}} /\right.$ $\left.R_{\mathrm{E}}\right)$, in the case of an axial quadrupole $(n=2)$. The value of the parameter $r_{2} / R_{\mathrm{E}}$ specifies indirectly (see Sect. 6.1) the corresponding maximum radial distance of each selected field line in the absence of the spherical magnetopause $\left(R_{\mathrm{M}} / R_{\mathrm{E}} \rightarrow \infty\right)$

\begin{tabular}{|c|c|c|c|c|c|c|c|}
\hline \multirow[t]{2}{*}{$r_{2} / R_{\mathrm{E}}$} & \multicolumn{7}{|l|}{$R_{\mathrm{M}} / R_{\mathrm{E}}$} \\
\hline & 10 & 25 & 50 & 100 & 250 & 500 & 1000 \\
\hline 10 & 7.2815 & 8.1499 & 8.1645 & 8.1650 & 8.1650 & 8.1650 & 8.1650 \\
\hline 25 & 9.5212 & 18.204 & 20.300 & 20.409 & 20.412 & 20.412 & 20.412 \\
\hline 50 & 9.8800 & 23.136 & 36.408 & 40.599 & 40.823 & 40.825 & 40.825 \\
\hline 500 & 9.9988 & 24.981 & 49.850 & 98.800 & 231.36 & 364.08 & 405.99 \\
\hline 1000 & 9.9997 & 24.995 & 49.963 & 99.700 & 245.31 & 462.72 & 728.15 \\
\hline $10^{6}$ & 10.000 & 25.000 & 50.000 & 100.00 & 250.00 & 500.00 & 1000.0 \\
\hline
\end{tabular}

Table 3. Maximum radial distances $\left(r_{m} / R_{\mathrm{E}}\right.$; (i) $\theta=90^{\circ}$ and (ii) $\left.\theta=39^{\circ} 13^{\prime} 53^{\prime \prime}, 140^{\circ} 46^{\prime} 07^{\prime \prime}\right)$ of selected magnetic field lines, for various values of the radius of the perfectly conducting spherical magnetopause $\left(R_{\mathrm{M}} / R_{\mathrm{E}}\right)$, in the case of an axial octupole $(n=3)$.
The value of the parameter $r_{3} / R_{\mathrm{E}}$ specifies indirectly (see Sect. 6.1) the corresponding maximum radial distance of each selected field line in the absence of the spherical magnetopause $\left(R_{\mathrm{M}} / R_{\mathrm{E}} \rightarrow \infty\right)$

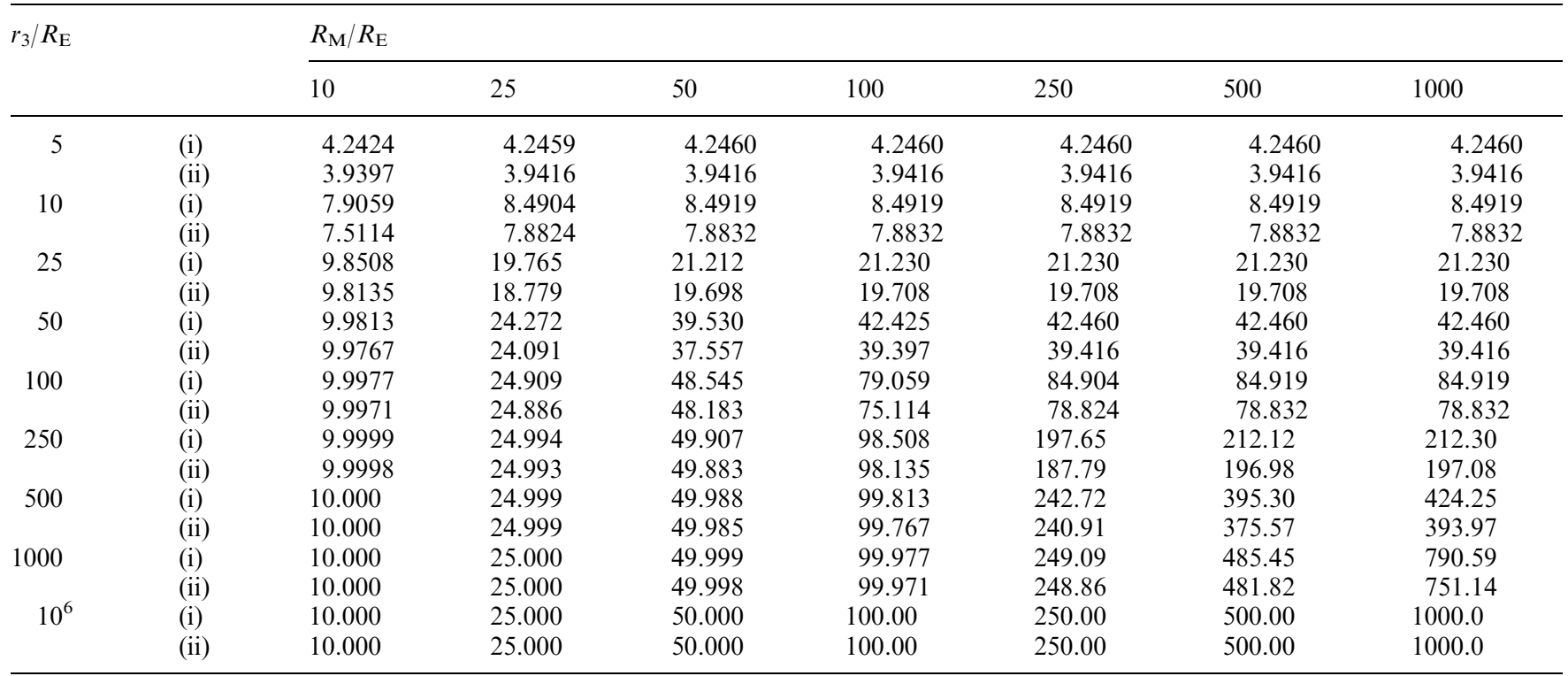


Table 4. Maximum radial distances $\left(r_{m} / R_{\mathrm{E}}\right.$; (i) $\theta=70^{\circ} 07^{\prime} 27^{\prime \prime}$, $109^{\circ} 52^{\prime} 33^{\prime \prime}$ and (ii) $\theta=30^{\circ} 33^{\prime} 20^{\prime \prime}, 149^{\circ} 26^{\prime} 40^{\prime \prime}$ ) of selected magnetic field lines, for various values of the radius of the perfectly conducting spherical magnetopause $\left(R_{\mathrm{M}} / R_{\mathrm{E}}\right)$, in the case of an axial sedecimupole $(n=4)$. The value of the parameter $r_{4} / R_{\mathrm{E}}$ specifies indirectly (see Sect. 6.1) the corresponding maximum radial distance of each selected field line in the absence of the spherical magnetopause $\left(R_{\mathrm{M}} / R_{\mathrm{E}} \rightarrow \infty\right)$

\begin{tabular}{|c|c|c|c|c|c|c|c|c|}
\hline$r_{4} / R_{\mathrm{E}}$ & & \multicolumn{7}{|l|}{$R_{\mathrm{M}} / R_{\mathrm{E}}$} \\
\hline \multirow[t]{2}{*}{5} & (i) & 4.2471 & 4.2475 & 4.2475 & 4.2475 & 4.2475 & 4.2475 & 4.2475 \\
\hline & (ii) & 3.9395 & 3.9398 & 3.9398 & 3.9398 & 3.9398 & 3.9398 & 3.9398 \\
\hline \multirow[t]{2}{*}{10} & (i) & 8.1402 & 8.4950 & 8.4951 & 8.4951 & 8.4951 & 8.4951 & 8.4951 \\
\hline & (ii) & 7.6878 & 7.8795 & 7.8795 & 7.8795 & 7.8795 & 7.8795 & 7.8795 \\
\hline 25 & (i) & 9.9454 & 20.350 & 21.235 & 21.238 & 21.238 & 21.238 & 21.238 \\
\hline 50 & (ii) & 9.9954 & 24.550 & 38.439 & 39.395 & 39.398 & 39.398 & 39.398 \\
\hline \multirow[t]{2}{*}{100} & (i) & 9.9998 & 24.979 & 49.334 & 81.402 & 84.950 & 84.951 & 84.951 \\
\hline & (ii) & 9.9997 & 24.972 & 49.100 & 76.879 & 78.795 & 78.795 & 78.795 \\
\hline \multirow[t]{2}{*}{250} & (i) & 10.000 & 25.000 & 49.983 & 99.454 & 203.50 & 212.35 & 212.38 \\
\hline & (ii) & 10.000 & 24.999 & 49.977 & 99.262 & 192.20 & 196.98 & 196.99 \\
\hline 500 & (i) & 10.000 & 25.000 & 49.999 & 99.966 & 246.67 & 407.01 & 424.71 \\
\hline $10^{6}$ & (ii) & 10.000 & 25.000 & 50.000 & 100.00 & 250.00 & 500.00 & 1000.0 \\
\hline
\end{tabular}

$(n=1)$ and indirectly $(2 \leq n \leq 4)$ in the captions to Tables 1-4. However, with the present definition, the values in each of the final columns $\left(R_{\mathrm{M}} / R_{\mathrm{E}}=1000\right)$ of Tables 1-4 are good approximations to the corresponding limiting values of the parameter $r_{m} / R_{\mathrm{E}}$ as $R_{\mathrm{M}} / R_{\mathrm{E}} \rightarrow \infty$ if $r_{n} / R_{\mathrm{E}} \ll R_{\mathrm{M}} / R_{\mathrm{E}}(1 \leq n \leq 4)$. The radial compression of magnetic field lines arises from the presence of the term $\left[1-\left(r / R_{\mathrm{M}}\right)^{2 n+1}\right]^{1 / n}$ in Eq. (14).

The numerical values of $r_{m} / R_{\mathrm{E}}$ presented in Tables 14 illustrate several important physical properties of a zonal magnetic multipole $(1 \leq n \leq 4)$ confined by a perfectly conducting (concentric) spherical magnetopause. Magnetic field lines that would extend to very large distances (i.e. $r_{n} / R_{\mathrm{E}}=10^{6}$ ) in the absence of the magnetopause $\left(R_{\mathrm{M}} / R_{\mathrm{E}} \rightarrow \infty\right)$ do indeed lie extremely close to the spherical magnetopause for a large, but finite, range of magnetopause radii $\left(10 \leq R_{\mathrm{M}} / R_{\mathrm{E}} \leq 1000\right)$. This particular subset of the numerical results presented in Tables $1-4$ corroborates the theoretical argument advanced at the end of Sect. 3. Moreover, magnetic field lines in the outer magnetosphere are greatly compressed by the presence of the perfectly conducting spherical magnetopause, as is intuitively obvious on physical grounds. Similarly, magnetic field lines in the inner magnetosphere are not greatly compressed by the presence of the magnetopause.

The use of terms such as "inner" and "outer", to describe regions of the compressed magnetosphere, requires some clarification. For present purposes, the outer magnetosphere can be defined accurately by the condition $r_{n} / R_{\mathrm{E}} \gg R_{\mathrm{M}} / R_{\mathrm{E}}$ and approximately by the condition $r_{n} / R_{\mathrm{E}} \geq R_{\mathrm{M}} / R_{\mathrm{E}}$. Likewise, the inner magnetosphere can be defined accurately by the condition $r_{n} / R_{\mathrm{E}} \ll R_{\mathrm{M}} / R_{\mathrm{E}}$ and approximately by the condition $r_{n} / R_{\mathrm{E}}<R_{\mathrm{M}} / R_{\mathrm{E}}$. These two approximate conditions are adequately accurate for the actual values of the two parameters (i.e. $r_{n} / R_{\mathrm{E}}$ and $R_{\mathrm{M}} / R_{\mathrm{E}}$ ) presented in Tables $1-4$.

The relative compression of a zonal magnetic field, caused by the presence of the perfectly conducting spherical magnetopause, decreases as $n$ increases. The relative compression is defined by the ratio $\left(r_{n}-r_{m}\right) / r_{n}$ for the particular field line that satisfies the ("initial") condition $r_{n}=R_{\mathrm{M}}$. With this definition, the percentage compressions are as follows $(1 \leq n \leq 4): 31.8 \%(n=1)$; $27.2 \%(n=2)$; (i) $20.9 \%$, (ii) $24.9 \%(n=3)$; (i) $18.6 \%$, (ii) $23.1 \%(n=4)$.

\subsection{Symmetric sectorial multipoles $(m=n)$}

Figure $3 \mathrm{a}-\mathrm{d}$ shows the magnetic-field-line configurations in one quadrant of the Northern Hemisphere $(0 \leq \theta \leq \pi / 2)$ for the first four low-degree symmetric sectorial magnetic multipoles (see Sect. 5.2); namely, a dipole $(m=n=1)$, a quadrupole $(m=n=2)$, an octupole $(m=n=3)$ and a sedecimupole $(m=n=4)$, each of which lies in the equatorial plane. These four magnetic-field-line configurations have been calculated using Eqs. (27), (28) and (29) for the illustrative case in which $R_{\mathrm{M}}=10 R_{\mathrm{E}}$. The numerical procedure employed to determine these magnetic-field-line configurations is an obvious extension of the numerical procedure described in detail for zonal multipoles (see Sect. 6.1). In practice, this procedure depends on the selection of suitable sets of values of the parameters $r_{n}^{n}, K_{n}^{n}$ and $\left(r_{n}^{n}\right)^{\prime}$, which occur in Eqs. (27), (28) and (29), respectively.

The bold (thick) continuous curves depict magnetopause field lines lying on the idealized spherical magnetopause surface $\left(r=R_{\mathrm{M}}\right)$, whereas the faint (thin) continuous curves and dotted curves depict planar 
Table 5. Positions of all neutral points $(P)$ and neutral rings $(R)$ on the spherical magnetopause surface $\left(r=R_{\mathrm{M}}\right)$ for the three special types of magnetic multipole. These are: (1) zonal, or axisymmetric, multipoles $(m=0)$; $(2)$ symmetric sectorial multipoles $(m=n)$; and (3) antisymmetric sectorial multipoles $(m=n-1)$. The positions of neutral points and rings are defined by co-latitude $(\theta)$ in the case of axisymmetric multipoles. The positions of neutral points are defined by both co-latitude and longitude $(\theta, \phi)$ in the case of non-axisymmetric multipoles. Longitude $\phi$ is measured relative to the appropriate phase angle $\left(\phi_{n}^{n}, \phi_{n}^{n-1}\right)$, as for Figs. 3 and 4 . A preceding italic letter is used to distinguish between neutral points $(P)$ and rings $(R)$. For complete clarity, a further distinction is made between an "isolated" neutral point $(P)$ on the spherical magnetopause surface and a "nonisolated" neutral point $(L)$ arising from the intersection of a neutral line with the spherical magnetopause

\begin{tabular}{|c|c|c|c|c|}
\hline Type of multipole & \multicolumn{4}{|c|}{ Degree of multipole } \\
\hline Zonal or axisymmetric & $\begin{array}{l}P(0) \\
P(\pi)\end{array}$ & $\begin{array}{l}P(0) \\
R(\pi / 2) \\
P(\pi)\end{array}$ & $\begin{array}{l}P(0) \\
R(\arccos \sqrt{1 / 5}) \\
R(\pi-\arccos \sqrt{1 / 5}) \\
P(\pi)\end{array}$ & $\begin{array}{l}P(0) \\
R(\arccos \sqrt{3 / 7}) \\
R(\pi / 2) \\
R(\pi-\arccos \sqrt{3 / 7}) \\
P(\pi)\end{array}$ \\
\hline
\end{tabular}

magnetospheric field lines lying in the equatorial plane and in one of the special meridional planes, respectively (see Sect. 5.2). Similar magnetic-field-line configurations exist in each quadrant of both the Northern and Southern Hemispheres. Thus the four magneticfield-line configurations presented in Fig. 3a-d illustrate the fact that the polar axis $(\theta=0, \pi)$ is a neutral line if $n>1$, as noted in Sect. 5.2. Therefore, if $n>1$, neutral points inevitably exist on the spherical magnetopause surface at the two poles. In addition, the four magnetic-field-line configurations presented in Fig. 3ad imply that $2 n$ equatorial neutral points exist on the spherical magnetopause surface, as also noted in Sect. 5.2. Table 5 lists, for the first four symmetric sectorial multipoles, the precise positions of the neutral points that lie on the spherical magnetopause surface. In this table, a distinction is made between an "isolated" neutral point $(P)$ and a "non-isolated" neutral point $(L)$ arising from the intersection of a (magnetospheric) neutral line with the spherical magnetopause. A network of magnetic field lines lying on the spherical magnetopause surface links all these neutral points and this network clearly becomes increasingly complex as $n$ increases.

In general, the idealized spherical magnetosphere comprises a "northern" portion separated from a "southern" portion by the equatorial plane $(\theta=\pi / 2)$, as illustrated in Fig. 3a-d. However, the distinction between "northern" and "southern" portions of the magnetosphere is spurious in the degenerate case $m=n=1$ (Fig. 3a), which corresponds to a magnetic dipole with its axis lying in the equatorial plane. The magnetic field lines are locally parallel to the equatorial plane for all values of $n$, as is clear from Fig. 3a-d (see Sect. 5.2). Moreover, the magnetosphere is essentially divided into $2 n$ azimuthal "segments" if $n>1$. 


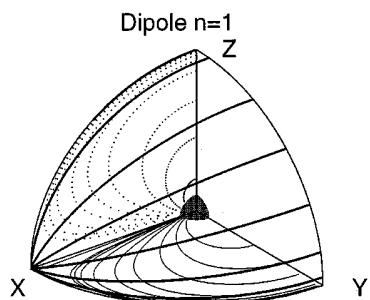

a)

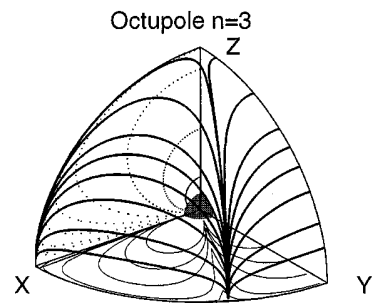

Fig. 3a-d. Illustrative magnetic-field-line configurations for lowdegree symmetric sectorial magnetic multipoles $(m=n)$ : a dipole $(n=1)$, b quadrupole $(n=2)$, c octupole $(n=3)$, and d sedecimupole $(n=4)$. The bold (thick) continuous curves depict magnetopause field lines lying on the idealized spherical magnetopause surface $\left(r=R_{\mathrm{M}}\right)$, the faint (thin) continuous curves depict magnetospheric field lines lying in the equatorial plane and the dotted curves depict magnetospheric field lines lying in one of the special meridional planes

As noted in Sect. 5.2, the magnetic field of a symmetric sectorial multipole is purely meridional $\left(B_{\phi}=0\right)$ in the $2 n$ meridional planes $\phi=\phi_{n}^{n}+k \pi / n$ $(k=0,1,2, \ldots, 2 n-1)$. In deriving Figs $3 \mathrm{a}-\mathrm{d}$, the values of the phase angle $\phi_{n}^{n}(1 \leq n \leq 4)$ have been chosen conveniently to give planar field lines in the plane $y=0$ of a system of Cartesian axes $\mathrm{O}(x, y, z)$. These axes have origin $\mathrm{O}$ at the centre of the Earth and $z$-axis coincident with the polar axis of the set of spherical polar coordinates $(r, \theta, \phi)$ defined in Sect. 2. The field lines in the plane $y=0$ are shown as dotted curves in Fig. $3 \mathrm{a}-\mathrm{d}$.

\subsection{Antisymmetric sectorial multipoles $(m=n-1)$}

Figure $4 \mathrm{a}-\mathrm{d}$ shows the magnetic-field-line configurations in one quadrant of the Northern Hemisphere $(0 \leq \theta \leq \pi / 2)$ for the first four low-degree antisymmetric sectorial magnetic multipoles (see Sect. 5.3); namely, a dipole $(m=0, n=1)$, a quadrupole $(m=1, n=2)$, an octupole $(m=2, n=3)$ and a sedecimupole $(m=3, n=4)$, each of which is aligned with the polar axis. These four magnetic-field-line configurations have been calculated using Eqs. (37) and (39) for the illustrative case in which $R_{\mathrm{M}}=10 R_{\mathrm{E}}$. As in the case of symmetric sectorial multipoles, the numerical procedure employed to determine these magnetic-field-line configurations is an obvious extension of the numerical procedure described in detail for zonal multipoles (see Sect. 6.1). In practice, this procedure depends on the selection of suitable sets of values of the parameters $r_{n}^{n-1}$ and $K_{n}^{n-1}$, which occur in Eqs. (37) and (39), respectively.

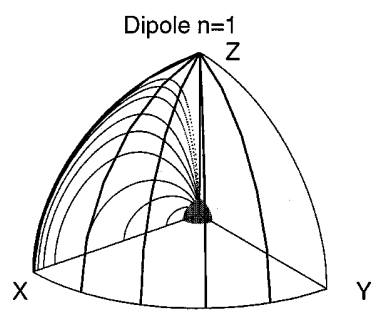

a)

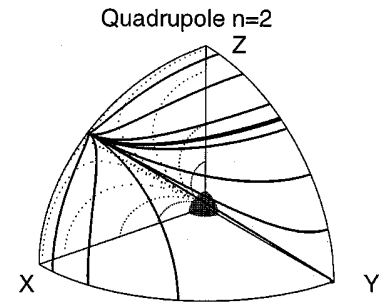

b)
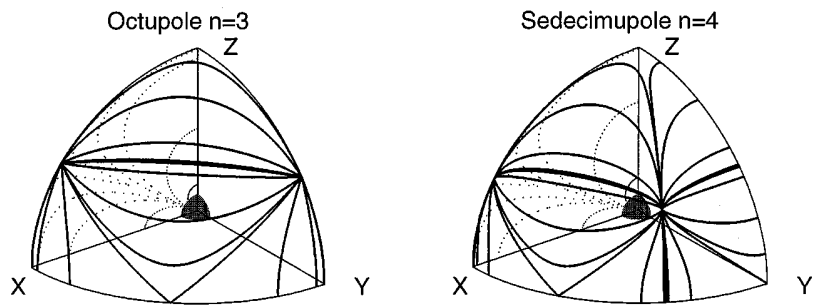

c)

d)

Fig. 4a-d. Illustrative magnetic-field-line configurations for lowdegree antisymmetric sectorial magnetic multipoles $(m=n-1)$ : a dipole $(n=1)$, b quadrupole $(n=2)$, c octupole $(n=3)$, and $\mathbf{d}$ sedecimupole $(n=4)$. The bold (thick) continuous curves depict magnetopause field lines lying on the idealized spherical magnetopause surface $\left(r=R_{\mathrm{M}}\right)$, and the dotted curves depict magnetospheric field lines lying in one of the special meridional planes

Once again, the bold (thick) continuous curves depict magnetopause field lines lying on the idealized spherical magnetopause surface $\left(r=R_{\mathrm{M}}\right)$, whereas the dotted curves depict planar magnetospheric field lines lying in one of the special meridional planes. Contrary to the case of a symmetric sectorial multipole $(m=n)$, no field lines lie in the equatorial plane of an antisymmetric sectorial multipole $(m=n-1)$, apart from the set of $2(n-1)$ equatorial neutral lines (see Sect. 5.3). As in Fig. 3a-d, similar magnetic-field-line configurations exist in each quadrant of both the Northern and Southern Hemispheres. Thus the four magnetic-fieldline configurations presented in Fig. $4 \mathrm{a}-\mathrm{d}$ illustrate the fact that $2(n-1)$ neutral lines exist in the equatorial plane and they also illustrate the fact that the polar axis $(\theta=0, \pi)$ is a neutral line if $n>2$, as noted in Sect. 5.3. Therefore, if $n>2$, neutral points inevitably exist on the spherical magnetopause surface at the two poles and also at the intersections of the $2(n-1)$ neutral lines in the equatorial plane with this surface. In addition, the four magnetic-field-line configurations presented in Fig. $4 \mathrm{a}-\mathrm{d}$ also implies that a further $4(n-1)$ neutral points exist on the two circles defined by the intersections of the circular conical surface $\theta=\arctan$ $\left[(n-1)^{1 / 2}\right], \theta=\pi-\arctan \left[(n-1)^{1 / 2}\right]$ with the spherical magnetopause surface, as also noted in Sect. 5.3. In this case there are $2(n-1)$ neutral points in each hemisphere. Table 5 lists, for the first four antisymmetric sectorial multipoles, the precise positions of the neutral points that lie on the spherical magnetopause surface. Once again, a network of magnetic field lines lying on the spherical magnetopause surface links all these neutral points and this network again becomes increasingly complex as $n$ increases. 
In general, the idealized spherical magnetosphere comprises an "equatorial" portion separated from northern and southern "polar" portions by the circular conical surface $\theta=\arctan \left[(n-1)^{1 / 2}\right], \quad \theta=\pi-\arctan$ $\left[(n-1)^{1 / 2}\right]$, as illustrated in Fig. 4a-d. However, the "polar" portions of the magnetosphere vanish in the degenerate case $m=0, n=1$ (Fig. 4a), which corresponds to a magnetic dipole aligned with the polar axis (see Fig. 2a). Moreover, all magnetic field lines that define the "equatorial" portion of the magnetosphere are locally perpendicular to the equatorial plane $(\theta=\pi / 2)$ if they cross it at a point where the magnetic field does not vanish (i.e. $|\mathbf{B}| \neq 0$ ), as is also clear from Fig. 4a-d (see Sect. 5.3).

As noted in Sect. 5.3, the magnetic field of an antisymmetric sectorial multipole is purely meridional $\left(B_{\phi}=0\right) \quad$ in the $2(n-1)$ meridional planes $\phi=\phi_{n}^{n-1}+k \pi /(n-1)(k=0,1,2, \ldots, 2 n-3)$. In deriving Fig. $4 \mathrm{a}-\mathrm{d}$, the values of the phase angle $\phi_{n}^{n-1}(1 \leq n \leq 4)$ have again been chosen conveniently to give planar field lines in the plane $y=0$ of a system of Cartesian axes $\mathrm{O}(x, y, z)$. The field lines in the plane $y=0$ are shown as dotted curves in Fig. $4 \mathrm{a}-\mathrm{d}$.

\section{Conclusions}

The main goal of this study is to consider possible, albeit ideal, configurations of the magnetic field in the outer magnetosphere during geomagnetic polarity reversals. This goal is achieved by considering the idealized problem of a magnetic multipole of order $m$ and degree $n$ located at the centre of a spherical cavity surrounded by a boundless perfect diamagnetic medium, as illustrated schematically in Fig. 1. In this idealization, the fixed spherical (magnetopause) boundary layer behaves as a perfectly conducting surface that shields the external diamagnetic medium from the compressed multipole magnetic field within the spherical (magnetosphere) cavity. The scientific reason for investigating such highly idealized models of the transitional magnetic field is to provide a sound theoretical framework for detailed studies of the nature of magnetospheric, ionospheric, auroral and cosmic-ray physics during geomagnetic polarity reversals.

The characteristic configurations of magnetic field lines in the outer magnetosphere are investigated for three special cases of an individual magnetic multipole confined by a perfectly conducting (concentric) spherical magnetopause. These special cases are (1) zonal multipoles $(m=0) ; \quad(2)$ symmetric sectorial multipoles $(m=n)$; and (3) antisymmetric sectorial multipoles $(m=n-1)$. Figure $2 \mathrm{a}-\mathrm{d}$ shows the magnetic-field-line configurations for the first four zonal magnetic multipoles $(1 \leq n \leq 4)$. In these (and subsequent) illustrative configurations, the radius of the spherical magnetopause is taken to be ten times the radius of the Earth (i.e. $\left.R_{\mathrm{M}}=10 R_{\mathrm{E}}\right)$. However, Tables $1-4$ illustrate the relative compression of representative magnetic field lines for a wide range of magnetopause radii $\left(10 \leq R_{\mathrm{M}} /\right.$ $\left.R_{\mathrm{E}} \leq 1000\right)$. In addition, Table 5 lists the precise positions of the neutral points and neutral rings that lie on the spherical magnetopause surface.

It is clear from the magnetic-field-line configurations presented in Fig. 2a-d that the idealized magnetosphere associated with a confined zonal magnetic multipole of arbitrary degree $n$ comprises $n$ separate ("self-contained") magnetic regions. A detailed discussion of auroral, ionospheric and magnetospheric physics in such "generalized magnetospheres", which might conceivably occur during the transition interval of a geomagnetic polarity reversal, is well beyond the intended scope of the present work. It should be noted briefly, however, that the actual existence of such idealized magnetospheres would necessarily imply multiple $(n+1)$ "magnetospheric cusps", and hence multiple $(n+1)$ "auroral-precipitation regions", as well as multiple $(n)$ "ring currents". Even more complex magnetospheres might well arise in the case of a linear combination of zonal multipoles (see Appendix B).

Figure $3 \mathrm{a}-\mathrm{d}$ shows the magnetic-field-line configurations in one quadrant of the Northern Hemisphere for the first four symmetric sectorial magnetic multipoles $\left(m=n ; 1 \leq n \leq 4 ; R_{\mathrm{M}}=10 R_{\mathrm{E}}\right)$. Table 5 lists the precise positions of the neutral points that lie on the spherical magnetopause surface. In the case of a symmetric sectorial sedecimupole $(m=n=4)$, there are 10 neutral points on the magnetopause surface. Two neutral points arise from the intersection of the polar neutral line with this surface and eight equally spaced neutral points lie in the equatorial plane. For both symmetric and antisymmetric sectorial magnetic multipoles (Figs. 3, 4), a network of magnetic field lines lying on the spherical magnetopause surface links all the neutral points. This network clearly becomes increasingly complex as $n$ increases.

Figure $4 \mathrm{a}-\mathrm{d}$ shows the magnetic-field-line configurations in one quadrant of the Northern Hemisphere for the first four antisymmetric sectorial magnetic multipoles $\left(m=n-1 ; 1 \leq n \leq 4 ; R_{\mathrm{M}}=10 R_{\mathrm{E}}\right)$. Table 5 lists the precise positions of the neutral points that lie on the spherical magnetopause surface. In the case of an antisymmetric sectorial sedecimupole $(m=3, n=4)$, there are 20 neutral points on the magnetopause surface. Eight neutral points arise from the intersection of polar and equatorial neutral lines with this spherical surface; two are at the poles and six are equally spaced in the equatorial plane. Six equally spaced neutral points lie on each of the two circles defined by the intersection of the circular conical surface $\theta=60^{\circ}, 120^{\circ}$ with the spherical magnetopause.

The existence of such an idealized (and possibly hypothetical) "antisymmetric sedecimupole magnetosphere" during the transition interval of a geomagnetic polarity reversal would almost inevitably result in a rich variety of auroral, ionospheric and magnetospheric phenomena. Moreover, in the case of both symmetric and antisymmetric sectorial multipoles, detailed research would be required to investigate the novel magnetospheric processes that are likely to arise from the existence of magnetic neutral lines that extend from the magnetopause to the surface of the Earth. 
A completely general property of the magnetic field just inside the spherical magnetopause in the case of a general magnetic multipole of degree $n$ (see Appendix A) may be stated as follows. The non-radial components of magnetic induction $\left(B_{\theta}, B_{\phi}\right)$ just inside the perfectly conducting spherical magnetopause $\left(r=R_{\mathrm{M}}\right)$ are increased by the factor $\{1+[(n+1) / n]\}$ relative to their corresponding values in the absence of the perfectly conducting spherical magnetopause $\left(R_{\mathrm{M}} \rightarrow \infty\right)$. In the case $n=1(m=0$ or $m=1)$, the non-radial components of magnetic induction $\left(B_{\theta}, B_{\phi}\right)$ are trebled by the presence of the perfectly conducting spherical magnetopause, as noted implicitly by $\mathrm{Wu}$ and Cole (1984a) for the case $m=0$. In the limit, as $n \rightarrow \infty$, the non-radial components of magnetic induction are doubled.

Finally, mention should be made of two properties of magnetic multipoles that remain totally unchanged by the introduction of the perfectly conducting (concentric) spherical magnetopause. First, magnetic field lines that are confined to special meridional planes or the equatorial plane, when the multipole is in free space, continue to be confined to these same planes when the perfectly conducting magnetopause is introduced. Although this result is axiomatic for the magnetic fields of axisymmetric (zonal) multipoles, it is perhaps slightly less obvious that the magnetic fields of non-axisymmetric multipoles are compressed by the perfectly conducting spherical magnetopause in such a way that planar field lines experience no torsion. Second, magnetic field lines that are perpendicular to certain meridional planes or the equatorial plane (everywhere except possibly at a set of neutral lines), when the multipole is in free space, continue to be perpendicular to these same planes when the perfectly conducting magnetopause is introduced.

Therefore, there exist classes of planar chargedparticle trajectories, which are confined to those planes (either meridional or equatorial) that are everywhere perpendicular to the magnetic field (B). Störmer (1955) obtained an exact equation for the radius of curvature of the trajectory of a charged particle whose orbital motion is confined to the equatorial plane of a magnetic dipole in free space. He found that every equatorial trajectory has the remarkable geometrical property that its radius of curvature at any point is proportional to the cube of its (equatorial) distance from the magnetic dipole. Moreover, Willis et al. (1997) extended Störmer's (1955) result by deriving equally remarkable exact equations for the radii of curvature of all possible planar charged-particle trajectories in an individual static magnetic multipole of arbitrary degree $(n)$ and order $(m)$, which is located in free space. An important conclusion of the present study is that these earlier results could be extended still further by deriving exact equations for the radii of curvature of all possible planar charged-particle trajectories in an individual static magnetic multipole confined by a concentric perfectly conducting spherical magnetopause. However, a detailed investigation of planar charged-particle trajectories in compressed multipole magnetic fields is beyond the intended scope of the present work, which is concerned exclusively with the magnetospheric magnet- ic-field-line configurations of confined magnetic multipoles.

Acknowledgements. The research reported was initiated while $\mathrm{ACH}$ pursued a twelve-month period of Industrial Training at the Rutherford Appleton Laboratory as part of the BSc Honours Degree Course in Mathematics at Coventry University. The authors thank a referee for particularly constructive and helpful comments, which have resulted in substantial improvements to the text and also the general results derived in the two appendices.

Topical Editor K.-H. Glassmeier thanks J. Vogt for his help in evaluating this paper.

\section{Appendix A}

\section{The general magnetic multiple of degree $n$}

It follows from Eq. (5) that the magnetic scalar potential $\left(\tilde{V}_{n}\right)$ of the general magnetic multipole of degree $n$ can be expressed in the form

$$
\tilde{V}_{n}=R_{\mathrm{E}}\left(R_{\mathrm{E}} / r\right)^{n+1}\left\{1+[(n+1) / n]\left(r / R_{\mathrm{M}}\right)^{2 n+1}\right\} Y_{n}(\theta, \phi) \text {, }
$$

where

$Y_{n}(\theta, \phi)=\sum_{m=0}^{n}\left(g_{n}^{m} \cos m \phi+h_{n}^{m} \sin m \phi\right) P_{n}^{m}(\cos \theta)$.

The function $Y_{n}(\theta, \phi)$ denotes an arbitrary linear combination of spherical harmonics of degree $n$. As noted in Sect. 2.2, the presence (absence) of the tilde is used as a convenient abbreviation that signifies the presence (absence) of the perfectly conducting spherical magnetopause. With this nomenclature,

$V_{n}=R_{\mathrm{E}}\left(R_{\mathrm{E}} / r\right)^{n+1} Y_{n}(\theta, \phi)$

represents the corresponding potential of the same multipole in free space $\left(R_{\mathrm{M}} \rightarrow \infty\right)$.

For brevity, the following definitions are introduced:

$\rho_{n}(r)=\left[1-\left(r / R_{\mathrm{M}}\right)^{2 n+1}\right]$

and

$\tau_{n}(r)=\left\{1+[(n+1) / n]\left(r / R_{\mathrm{M}}\right)^{2 n+1}\right\}$.

It is then straightforward to show that

$\partial \tilde{V}_{n} / \partial r=-(n+1)\left(R_{\mathrm{E}} / r\right)^{n+2} \rho_{n} Y_{n}=\rho_{n} \partial V_{n} / \partial r$,

$\partial \tilde{V}_{n} / r \partial \theta=\left(R_{\mathrm{E}} / r\right)^{n+2} \tau_{n} \partial Y_{n} / \partial \theta=\tau_{n} \partial V_{n} / r \partial \theta$,

and

$$
\begin{aligned}
\partial \tilde{V}_{n} / r \sin \theta \partial \phi & =\left(R_{\mathrm{E}} / r\right)^{n+2}\left(\tau_{n} / \sin \theta\right) \partial Y_{n} / \partial \phi \\
& =\tau_{n} \partial V_{n} / r \sin \theta \partial \phi .
\end{aligned}
$$

Therefore, the relationships between the magnetic-field components $\left(\tilde{B}_{r, n}, \tilde{B}_{\theta, n}, \tilde{B}_{\phi, n}\right)$ and $\left(B_{r, n}, B_{\theta, n}, B_{\phi, n}\right)$ are as follows $(\mathbf{B}=-\operatorname{grad} V)$ :

$\tilde{B}_{r, n}=\rho_{n} B_{r, n}=\left[1-\left(r / R_{\mathrm{M}}\right)^{2 n+1}\right] B_{r, n}$, 
$\tilde{B}_{\theta, n}=\tau_{n} B_{\theta, n}=\left\{1+[(n+1) / n]\left(r / R_{\mathrm{M}}\right)^{2 n+1}\right\} B_{\theta, n}$

$\tilde{B}_{\phi, n}=\tau_{n} B_{\phi, n}=\left\{1+[(n+1) / n]\left(r / R_{\mathrm{M}}\right)^{2 n+1}\right\} B_{\phi, n}$.

Consequently, Eqs. (6), (7) and (8) are valid for the general magnetic multipole of degree $n$ (which involves summation over $m ; 0 \leq m \leq n)$ and not just for an individual zonal, symmetric sectorial or antisymmetric sectorial multipole of degree $n$.

\section{Appendix B}

Exact equation for the magnetic field lines

of a linear combination of zonal multipoles

As noted briefly in Sect. 3, it is possible to generalize Eq. (14) to the case of an arbitrary linear combination of zonal, or axisymmetric, multipoles. This generalization can be derived elegantly by analogy with an equivalent problem in hydrodynamics (Lamb, 1945). The analogy depends on the fact that exact stream-line equations for incompressible irrotational fluids correspond to exact field-line equations for current-free magnetic fields (for which curl $\mathbf{B}=0, \mathbf{B}=-\operatorname{grad} V$ and $\nabla^{2} V=0$, using the nomenclature introduced in Sect. 2).

For an incompressible irrotational fluid, the velocity potential, $\Phi$, satisfies the continuity equation $\nabla^{2} \Phi=0$ (Lamb, 1945). An important method of solving this equation is the one based on the use of spherical harmonics; this method is especially suitable if the boundary conditions have to be applied at spherical surfaces. The classical problem in hydrodynamics of a multiple source (multipole) at the origin has been considered by Lamb (1945; Section 95). The appropriate equations for an individual zonal multipole in free space are

$\Phi_{n}=r^{-n-1} P_{n}^{0}(\mu), \quad \Psi_{n}=-\left(r^{-n} / n\right)\left(1-\mu^{2}\right) \mathrm{d} P_{n}^{0}(\mu) / \mathrm{d} \mu$,

where $\Phi_{n}$ denotes the velocity potential (magnetic scalar potential) of the zonal multipole of degree $n, P_{n}^{0}(\mu)$ is the corresponding Legendre polynomial, $\mu=\cos \theta$ and $\Psi_{n}$ denotes the associated stream function. The stream (field) lines are then given by $\Psi=$ constant (in all meridional planes).

Exact stream (field) lines in the case of an arbitrary linear combination of zonal multipoles can be determined easily with the aid of the stream function concept. If the velocity potential is of the general form

$\Phi=\sum_{n} a_{n} \Phi_{n}$,

the corresponding stream function is given by

$\Psi=\sum_{n} a_{n} \Psi_{n}$ and the exact stream (field) line equation is $\Psi=$ constant. This result was first published by Backus (1988).

The results presented by Lamb (1945; Section 95) also provide an ingenious means of deriving exact fieldline equations for both a single zonal multipole and an arbitrary linear combination of zonal multipoles in the presence of a perfectly conducting spherical surface. In particular, Lamb also considered inverse zonal multipoles of arbitrary degree $n$. The corresponding equations for the velocity potential and stream function of an inverse zonal multipole are

$\tilde{\Phi}_{n}=r^{n} P_{n}^{0}(\mu)$,

$\tilde{\Psi}_{n}=\left[r^{n+1} /(n+1)\right]\left(1-\mu^{2}\right) \mathrm{d} P_{n}^{0}(\mu) / \mathrm{d} \mu$.

Therefore, if the velocity potential is of the following general form

$\Phi=\sum_{n} a_{n} \Phi_{n}+\sum_{n} \tilde{a}_{n} \tilde{\Phi}_{n}$,

the general exact equation for stream (field) lines becomes

$\Psi=\sum_{n} a_{n} \Psi_{n}+\sum_{n} \tilde{a}_{n} \tilde{\Psi}_{n}=$ constant

The combined magnetic scalar potential of a single multipole of degree $n$ and its inverse counterpart is defined by Eq. (9) in Sect. 3. However, it should be noted that Eqs. (9) and (14) can be derived directly from Eqs. (B5) and (B6), respectively, by making the substitutions $a_{n}=g_{n}^{0} R_{\mathrm{E}}^{n+2}$ and $\tilde{a}_{n}=g_{n}^{0}[(n+1) / n]\left(R_{\mathrm{E}}^{n+2} / R_{\mathrm{M}}^{2 n+1}\right)$. More generally, Eq. (B6) provides an exact expression for the field lines of an arbitrary linear combination of zonal magnetic multipoles, confined by a perfectly conducting (concentric) spherical surface.

\section{References}

Backus, G. E., The field lines of an axisymmetric magnetic field, Geophys. J., 93, 413-417, 1988.

Beard, D. B., The solar wind geomagnetic field boundary, Rev. Geophys., 2, 335-365, 1964.

Beard, D. B., The solar wind, Rep. Prog. Phys., 30, 409-444, 1967.

Beyer, W. H., CRC standard mathematical tables, 27th Edn, CRC Press, Boca Raton, Florida, 1984.

Chapman, S., and J. Bartels, Geomagnetism, Vols I and II, Oxford University Press, London, 1940.

Jeffreys, B., Derivations of the equation for the field lines of an axisymmetric multipole, Geophys. J., 92, 355-356, 1988.

Lamb, H., Hydrodynamics (Sixth Edn), Dover Publications, New York, 1945

Matsushita, S., and W. H. Campbell, Physics of geomagnetic phenomena, Vol II (Appendix 3), Academic Press, New York, 1967.

Merrill, R. T., and P. L. McFadden, Geomagnetic polarity transitions, Rev. Geophys., 37, 201-226, 1999.

Price, A. T., Electromagnetic induction within the Earth, in Physics of geomagnetic phenomena, Vol I, Eds S. Matsushita and W. H. Campbell, Academic Press, New York, pp 235-298, 1967.

Roederer, J. G., Geomagnetic field distortions and their effects on radiation belt particles, Rev. Geophys. Space Phys., 10, 599-630, 1972. 
Störmer, C., The polar aurora, Oxford University Press, London, 1955.

Vestine, E. H., Main geomagnetic field, in Physics of geomagnetic phenomena, Vol I, Eds S. Matsushita and W. H. Campbell, Academic Press, New York, pp 181-234, 1967.

Willis, D. M., and A. R. Gardiner, Equations for the field lines of a sectorial magnetic multipole, Geophys. J., 95, 625-632, 1988.

Willis, D. M., and L. R. Young, Equation for the field lines of an axisymmetric magnetic multipole, Geophys. J. R. Astron. Soc., 89, 1011-1022, 1987.
Willis, D. M., A. R. Gardiner, V. N. Davda, and V. J. Bone, Planar charged-particle trajectories in multipole magnetic fields, Ann. Geophysicae, 15, 197-210, 1997.

Winch, D. E., The fourth order geomagnetic multipole: the sedecimupole, Pure Appl. Geophys., 67, 112-122, 1967.

Wu, Z.-J., and K. D. Cole, Magnetic field in a cavity of a perfect diamagnetic material, Planet. Space Sci., 32, 707-715, 1984a.

Wu, Z.-J., and K. D. Cole, Pressure comparison method in magnetopause shape calculation: under uniform outside pressure, Planet. Space Sci., 32, 1029-1034, 1984b. 